\title{
A MUSIC-type Algorithm for Detecting Internal Corrosion from Electrostatic Boundary Measurements *
}

\author{
Habib Ammari ${ }^{\dagger} \quad$ Hyeonbae Kang ${ }^{\ddagger} \quad$ Eunjoo Kim ${ }^{\ddagger} \quad$ Kaouthar Louati $^{\S}$ \\ Michael S. Vogelius
}

October 24, 2007

\begin{abstract}
We establish an asymptotic representation formula for the steady state current perturbations caused by internal corrosive boundary parts of small surface measure. Based on this formula we design a non-iterative method of MUSIC (multiple signal classification) type for localizing the corrosive parts from voltage-to-current observations. We perform numerical experiments to test the viability of the algorithm and the results clearly demonstrate that the algorithm works well even in the presence of relatively high noise ratios.
\end{abstract}

Mathematics subject classification (MSC2000): 35R30

Keywords: corrosion, asymptotic representation formula, reconstruction, layer potentials

\section{Introduction}

Hidden corrosion, for instance in pipes, is a serious problem that is responsible for significant economic losses and represents a dangerous threat to safety. In the field of nondestructive evaluation, new and improved techniques are therefore constantly being sought to facilitate the detection of such hidden corrosion.

Corrosion occurs in many different forms, and several mathematical models for electrostatic fields in the presence of corrosion have been studied in the literature (see, for example, Kaup and Santosa [16], Kaup et al. [17], Vogelius and Xu [21], Inglese [12], Luong and Santosa [13], Banks et al. [3] and references therein).

${ }^{*}$ H.A. is partially supported by the Brain Pool Korea Program at Seoul National University, H.K. is partially supported by KOSEF grant R01-2006-000-10002-0, E.K. is supported by BK21 Math. Division at Seoul National University, and M.S.V. is partially supported by NSF grant DMS-0604999.

${ }^{\dagger}$ Laboratoire Ondes et Acoustique, CNRS \& ESPCI, 10 rue Vauquelin, 75231 Paris Cedex 05, France (habib.ammari@polytechnique.fr).

${ }^{\ddagger}$ Department of Mathematical Sciences, Seoul National University, Seoul 151-747, Korea (hbkang@snu.ac.kr, kej@math.snu.ac.kr).

${ }^{\S}$ Centre de Mathématiques Appliquées, Ecole Polytechnique, 91128 Palaiseau Cedex, France (louati@cmapx.polytechnique.fr).

IDepartment of Mathematics, Rutgers University, New Brunswick, NJ 08903, USA (vogelius@math.rutgers.edu). 
In this paper, we adopt the potential model: Laplace's equation holds in the metal pipe, and the effect of corrosion is described by means of a linear Robin boundary condition. A model like this is motivated by a number of observations. On the one hand, corrosion tends to roughen a surface: this effect can be modelled by the introduction of a thin coating characterized by rapid oscillations. In the limit as the thickness of the coating goes to zero and the rapidity of the oscillations diverges, the emergence of a Robin boundary conditions has been shown by Buttazo and Kohn [5]. On the other hand, the study of active electrochemical corrosion processes can be based on "first principles", which conjecture a certain relation between the number of "free electrons" and the energy level (typically an exponential Boltzmann-type distribution). Potential models with exponential boundary conditions that arise from such considerations are often associated with the names of Butler and Volmer. Vogelius and Xu [21] study a potential model of this kind. If we linearize the boundary condition of this model, we get the linear Robin boundary condition studied here.

To set up the problem mathematically, consider a simply connected, bounded $\mathcal{C}^{2, \alpha}$ domain $U$ in $\mathbb{R}^{2}$ for some $0<\alpha \leq 1$, and a simply connected $\mathcal{C}^{2, \alpha}$ domain $D$, compactly contained in $U . \Omega=U \backslash \bar{D}$ represents the specimen to be inspected, e.g., the cross section of the pipe. We define $\Gamma_{e}=\partial U$ and $\Gamma_{i}=\partial D$, so that $\partial \Omega=\Gamma_{i} \cup \Gamma_{e}$. Suppose that the inaccessible boundary $\Gamma_{i}$ contains some corrosive, connected parts $I_{s}, s=1, \ldots, m$. The open curves $I_{s}$ are well-separated, i.e., there is a constant $c_{0}>0$ such that $\operatorname{dist}\left(I_{i}, I_{j}\right)>c_{0}$ for $i \neq j$, and the corrosion coefficient, $\gamma_{s} \in \mathcal{C}^{1}\left(I_{s}\right)$, of each $I_{s}, s=1, \ldots, m$, satisfies

$$
0 \leq \gamma_{s} \leq C_{0},
$$

for some constant $C_{0}$, and is not identically zero. Let

$$
\gamma(x)=\sum_{s=1}^{m} \gamma_{s} \chi_{s}(x), \quad x \in \Gamma_{i},
$$

where $\chi_{s}$ denotes the characteristic function of $I_{s}$. A typical shape of $\Omega$ would be an annulus. We assume that the curve length of each $I_{s}$ is small, to be specific,

$$
d_{0} \epsilon \leq\left|I_{s}\right|_{\Gamma_{i}} \leq D_{0} \epsilon, \quad s=1, \ldots, m,
$$

where $\epsilon$ is a small parameter, representing the common order of magnitude of $I_{s}$, and $d_{0}$, $D_{0}$ are positive constants. Each curve $I_{s}$ is centered around a fixed point $z_{s}$ :

$$
I_{s}=\left\{z \in \Gamma_{i}: d_{\Gamma_{i}}\left(z, z_{s}\right)<\left|I_{s}\right| / 2\right\} .
$$

Here $|\cdot|_{\Gamma_{i}}$ and $d_{\Gamma_{i}}(\cdot, \cdot)$ refer to the one-dimensional curve length on $\Gamma_{i}$. The internal voltage potential $u_{\epsilon}$, generated by a voltage $f$ applied to $\Gamma_{e}$, satisfies

$$
\begin{cases}\Delta u_{\epsilon}=0 & \text { in } \Omega, \\ -\frac{\partial u_{\epsilon}}{\partial \nu}+\gamma u_{\epsilon}=0 & \text { on } \Gamma_{i}, \\ u_{\epsilon}=f & \text { on } \Gamma_{e},\end{cases}
$$

where $\nu$ is the inward normal to $\Omega$ on $\Gamma_{i}$ (i.e., the outward normal to $D$ ).

A realistic inverse problem in corrosion detection consists of the determination of the corrosion damage on the inaccessible boundary part, $\Gamma_{i}$, when the available data are a finite 
number of voltage-to-current pairs on the accessible part $\Gamma_{e}$. The difficulties of this inverse problem stem from its inherent ill-posedness and nonlinearity. Most of the techniques for detecting the corrosion are based on iterative algorithms: least-square-fit algorithms and Newton-type iteration schemes. These methods typically incur tremendous computational costs to get close to the true corrosion damage, but more troubling: they frequently get "stuck" near local minima.

The purpose of this work is to design a direct (non-iterative) technique for electrostatic corrosion detection. This algorithm is of MUSIC-type (multiple signal classification) and is based on an accurate asymptotic representation formula for the steady state boundary currents. In many ways it is closely related to the algorithm developed in [4] for the purpose of detection of small internal defects.

The Cauchy data continuation technique, as studied in the paper by Yang, Choulli, and Cheng [22] for thermal detection of corrosion, is also a direct method. For reasons of instability of the solution of the Cauchy problem it is less accurate than the method described here. Furthermore it makes use of the formula $\gamma=\left(1 / u_{\epsilon}\right) \partial u_{\epsilon} / \partial \nu$ which, in the electrostatic case is somewhat "unfortunate", due to potential sign changes (and corresponding zeroes) of $u_{\epsilon}$. A significant ("signal-to noise") advantage of the present method is that it directly uses the boundary current perturbation data $\left.\left(\frac{\partial u_{\epsilon}}{\partial \nu}-\frac{\partial u_{0}}{\partial \nu}\right)\right|_{\Gamma_{e}}$ (as opposed to $\left.\left.\frac{\partial u_{\epsilon}}{\partial \nu}\right|_{\Gamma_{e}}\right)$. Here $u_{0}$ is the voltage potential in the absence of corrosion, i.e., the solution to

$$
\left\{\begin{array}{ll}
\Delta u_{0}=0 & \text { in } \Omega \\
-\frac{\partial u_{0}}{\partial \nu}=0 & \text { on } \Gamma_{i} \\
u_{0}=f & \text { on } \Gamma_{e}
\end{array} .\right.
$$

The present paper is organized as follows: In Section 2 we review some basic facts about layer potentials. In the following section we establish a representation formula for the unique solution to (1.4). This formula generalizes the formula proved by Kang and Seo in $[14,15]$. The main goal of Section 4 is to rigorously derive an asymptotic expansion for $\frac{\partial u_{\epsilon}}{\partial \nu}-\frac{\partial u_{0}}{\partial \nu}$ on $\Gamma_{e}$. Section 5 is then devoted to the design of the numerical algorithm, and several computational experiments with it.

\section{Layer Potentials}

In this section we review some well-known properties of layer potentials for the Laplacian, and establish some useful identities. Let $\Phi(x)$ be the fundamental solution associated with the Laplacian $\Delta$,

$$
\Phi(x)=\frac{1}{2 \pi} \ln |x|, \quad x \neq 0 .
$$

Let $D$ be a simply connected, bounded $\mathcal{C}^{2, \alpha}$ domain in $\mathbb{R}^{2}$, and let $\Gamma:=\partial D$. Let $H^{1}(D)$ denote the set of functions $f \in L^{2}(D)$ such that $\nabla f \in L^{2}(D)$. Furthermore, we define $H^{2}(D)$ as the space of functions $u \in H^{1}(D)$ such that $\partial^{2} u \in L^{2}(D)$, and the space $H^{3 / 2}(D)$ as the interpolation space $\left[H^{1}(D), H^{2}(D)\right]_{1 / 2}$. Let $H^{1 / 2}(\partial D)$ be the set of traces of functions in $H^{1}(D)$ and $H^{-1 / 2}(\partial D)=\left(H^{1 / 2}(\partial D)\right)^{*}$. Finally, let $H^{1}(\partial D)$ denote the set of functions $f \in L^{2}(\partial D)$ such that $\partial f / \partial \tau \in L^{2}(\partial D)$, where $\partial / \partial \tau$ is the tangential derivative. 
The single-layer and double-layer potentials of a function $\varphi \in L^{2}(\Gamma)$, denoted $\mathcal{S}_{\Gamma}[\varphi]$ and $\mathcal{D}_{\Gamma}[\varphi]$, respectively, are defined by

$$
\begin{aligned}
& \mathcal{S}_{\Gamma}[\varphi](x):=\int_{\Gamma} \Phi(x-y) \varphi(y) d \sigma_{y}, \quad x \in \mathbb{R}^{2}, \\
& \mathcal{D}_{\Gamma}[\varphi](x):=\int_{\Gamma} \frac{\partial}{\partial \nu_{y}} \Phi(x-y) \varphi(y) d \sigma_{y}, \quad x \in \mathbb{R}^{2} \backslash \Gamma .
\end{aligned}
$$

For a function $u$, defined in $\mathbb{R}^{2} \backslash \Gamma$, we denote

$$
\left.\frac{\partial u}{\partial \nu}\right|_{ \pm}(x):=\lim _{t \rightarrow 0^{+}}\left\langle\nabla u\left(x \pm t \nu_{x}\right), \nu_{x}\right\rangle, \quad x \in \Gamma
$$

if the limit exists. Here $\nu_{x}$ is the outward unit normal to $\Gamma=\partial D$ at $x$, and $\langle$,$\rangle denotes$ the scalar product in $\left.\mathbb{R}^{2} \cdot u\right|_{ \pm}$are defined likewise.

It is well-known (see for example [11]) that for $\varphi \in L^{2}(\Gamma)$

$$
\begin{gathered}
\left.\frac{\partial\left(\mathcal{S}_{\Gamma}[\varphi]\right)}{\partial \nu}\right|_{ \pm}(x)=\left( \pm \frac{1}{2} I+\mathcal{K}_{\Gamma}^{*}\right)[\varphi](x), \quad x \in \Gamma, \\
\left.\mathcal{D}_{\Gamma}[\varphi]\right|_{ \pm}(x)=\left(\mp \frac{1}{2} I+\mathcal{K}_{\Gamma}\right)[\varphi](x), \quad x \in \Gamma,
\end{gathered}
$$

where $\mathcal{K}_{\Gamma}$ is defined by

$$
\mathcal{K}_{\Gamma}[\varphi](x)=\frac{1}{2 \pi} \int_{\Gamma} \frac{\left\langle y-x, \nu_{y}\right\rangle}{|x-y|^{2}} \varphi(y) d \sigma_{y},
$$

and $\mathcal{K}_{\Gamma}^{*}$ is the $L^{2}$-adjoint of $\mathcal{K}_{\Gamma}$, i.e.,

$$
\mathcal{K}_{\Gamma}^{*}[\varphi](x)=\frac{1}{2 \pi} \int_{\Gamma} \frac{\left\langle x-y, \nu_{x}\right\rangle}{|x-y|^{2}} \varphi(y) d \sigma_{y} .
$$

Since $\Gamma$ is a $\mathcal{C}^{2, \alpha}$ curve, there is a constant $C$ such that

$$
\frac{\left|\left\langle x-y, \nu_{x}\right\rangle\right|}{|x-y|^{2}} \leq C, \quad x, y \in \Gamma
$$

Thus it immediately follows that $\mathcal{K}_{\Gamma}$ is bounded on $L^{p}(\Gamma)$ for $1 \leq p \leq \infty$. Moreover, the operator $\varphi \mapsto \nabla_{T} \mathcal{K}_{\Gamma}[\varphi]$, where $\nabla_{T}$ denotes the tangential derivative on $\Gamma$, is a CalderónZygmund operator on $\Gamma$ which is known to be bounded on $L^{p}(\Gamma)(1<p<\infty), \mathcal{C}^{\beta}(\Gamma)$ and $\mathcal{C}^{1, \beta}(\Gamma)(0<\beta<1)$ (see [19]). Therefore $\mathcal{K}_{\Gamma}$ and $\mathcal{K}_{\Gamma}^{*}$ map continuously $L^{p}(\Gamma)$ into $H^{1, p}(\Gamma)$, $\mathcal{C}^{\beta}(\Gamma)$ into $\mathcal{C}^{1, \beta}(\Gamma)(0<\beta<1)$, and $\mathcal{C}^{1, \beta}(\Gamma)$ into $\mathcal{C}^{2, \beta}(\Gamma)(0<\beta \leq \alpha)$ since $\Gamma$ is $\mathcal{C}^{2, \alpha}$. Here $H^{1, p}(\Gamma)$ is the collection of functions in $L^{p}(\Gamma)$ whose derivatives also belong to $L^{p}(\Gamma)$.

Lemma 2.1 The operators $\frac{1}{2} I+\mathcal{K}_{\Gamma}$ and $\frac{1}{2} I+\mathcal{K}_{\Gamma}^{*}$ are invertible on $L^{p}(\Gamma), 1<p$, as well as on $\mathcal{C}^{k}(\Gamma)$ for $k=1,2$.

Proofs of Lemma 2.1 on the spaces $L^{2}(\Gamma)$ and $\mathcal{C}(\Gamma)$ can be found in [11]. The invertibility on the other spaces can seen as follows: consider the equation

$$
\left(\frac{1}{2} I+\mathcal{K}_{\Gamma}\right)[u]=f \quad \text { or } \quad\left(\frac{1}{2} I+\mathcal{K}_{\Gamma}^{*}\right)[u]=f .
$$


If $f \in L^{p}(\Gamma)$ for $p>2$ and $u \in L^{2}(\Gamma)$, then $\mathcal{K}_{\Gamma} u \in L^{p}(\Gamma)$ and hence $u \in L^{p}(\Gamma)$. This shows that $\frac{1}{2} I+\mathcal{K}_{\Gamma}$ and $\frac{1}{2} I+\mathcal{K}_{\Gamma}^{*}$ are invertible on $L^{p}(\Gamma)$ for $p>2$. Invertibility on $L^{p}(\Gamma)$ for $1<p<2$ can be seen by duality. Similarly one can prove invertibility on $\mathcal{C}^{k}(\Gamma)$ for $k=1,2$.

In the case where $\Gamma$ is a circle of radius $r$

$$
\frac{\left\langle\nu_{x}, x-y\right\rangle}{|x-y|^{2}}=\frac{1}{2 r} \quad, \quad x, y \in \Gamma
$$

and hence

$$
\mathcal{K}_{\Gamma}[\varphi](x)=\mathcal{K}_{\Gamma}^{*}[\varphi](x)=\frac{1}{4 \pi r} \int_{\Gamma} \varphi(y) d \sigma_{y} .
$$

It now follows from (2.3) and (2.6) that

$$
\mathcal{S}_{\Gamma}[1](x)= \begin{cases}r \ln r & \text { if }|x| \leq r, \\ r \ln |x| & \text { if }|x| \geq r,\end{cases}
$$

in the case where $\Gamma$ is a circle of radius $r$.

Finally, we recall some boundedness properties of single layer potentials for later use. If $p>1$, there is a constant $C_{p}$ such that

$$
\left\|\mathcal{S}_{\Gamma}[\varphi]\right\|_{L^{\infty}(\Gamma)} \leq C_{p}\|\varphi\|_{L^{p}(\Gamma)}
$$

for all $\varphi \in L^{p}(\Gamma)$. In fact, (2.8) can be proved using Hölder's inequality. It is also well-known that

$$
\left\|\mathcal{S}_{\Gamma}[\varphi]\right\|_{H^{1}(D)} \leq C\|\varphi\|_{L^{2}(\Gamma)}
$$

for any $\varphi \in L^{2}(\Gamma)$.

\section{Representation Formula}

Recall that $\Omega=U \backslash \bar{D}$, where $U$ and $D$ are simply connected, bounded $\mathcal{C}^{2}$ domains. Let $\Gamma_{e}=\partial U$ and $\Gamma_{i}=\partial D$. For $f \in H^{1}\left(\Gamma_{e}\right)$, let $u_{0}$ be the solution in the absence of corrosion, i.e., the solution to the problem (1.5). We seek to represent $u_{0}$ in the following form

$$
u_{0}=\mathcal{D}_{\Gamma_{e}}\left[\varphi_{0}\right]+\mathcal{S}_{\Gamma_{i}}\left[\psi_{0}\right] \quad \text { in } \Omega
$$

for some functions $\varphi_{0} \in H^{1}\left(\Gamma_{e}\right)$ and $\psi_{0} \in L^{2}\left(\Gamma_{i}\right)$. The boundary conditions in (1.5) are, due to the relations (2.4) and (2.3), equivalent to

$$
\left(\begin{array}{cc}
\frac{1}{2} I+\mathcal{K}_{\Gamma_{e}} & \mathcal{S}_{\Gamma_{i}} \\
-\frac{\partial}{\partial \nu_{i}} \mathcal{D}_{\Gamma_{e}} & -\frac{1}{2} I-\mathcal{K}_{\Gamma_{i}}^{*}
\end{array}\right)\left(\begin{array}{l}
\varphi_{0} \\
\psi_{0}
\end{array}\right)=\left(\begin{array}{l}
f \\
0
\end{array}\right) \in H^{1}\left(\Gamma_{e}\right) \times L^{2}\left(\Gamma_{i}\right)
$$

where $\nu_{e}$ and $\nu_{i}$ denote outward normal $\nu$ to $\Omega$ on $\Gamma_{e}$ and $\Gamma_{i}$ (the subscripts $e$ and $i$ emphasize that they are defined on $\Gamma_{e}$ and $\Gamma_{i}$, respectively). For example, $\frac{\partial}{\partial \nu_{i}} \mathcal{D}_{\Gamma_{e}}[\varphi]=\nu_{i} \cdot \nabla \mathcal{D}_{\Gamma_{e}}[\varphi]$. 
Lemma 3.1 For $p>1$, let $X_{p}:=L^{p}\left(\Gamma_{e}\right) \times L^{p}\left(\Gamma_{i}\right)$ and

$$
A_{0}:=\left(\begin{array}{cc}
\frac{1}{2} I+\mathcal{K}_{\Gamma_{e}} & \mathcal{S}_{\Gamma_{i}} \\
-\frac{\partial}{\partial \nu_{i}} \mathcal{D}_{\Gamma_{e}} & -\frac{1}{2} I-\mathcal{K}_{\Gamma_{i}}^{*}
\end{array}\right) .
$$

Then $A_{0}$ is invertible on $X_{p}$, as well as on $H^{1}\left(\Gamma_{e}\right) \times L^{2}\left(\Gamma_{i}\right)$.

Proof. Since there is a distance between $\Gamma_{e}$ and $\Gamma_{i}$, the operator $A_{0}$ is a compact perturbation of

$$
\left(\begin{array}{cc}
\frac{1}{2} I+\mathcal{K}_{\Gamma_{e}} & 0 \\
0 & -\frac{1}{2} I-\mathcal{K}_{\Gamma_{i}}^{*}
\end{array}\right),
$$

which is known to be invertible on $X_{p}$ (Lemma 2.1). Therefore, it suffices, by applying the Fredholm alternative, to show that the operator $A_{0}$ is injective. Suppose that $(\varphi, \psi) \in X_{p}$ satisfies

$$
A_{0}\left(\begin{array}{l}
\varphi \\
\psi
\end{array}\right)=0
$$

Since $\left(\frac{1}{2} I+\mathcal{K}_{\Gamma_{e}}\right)[\varphi]=-\mathcal{S}_{\Gamma_{i}}[\psi]$ on $\Gamma_{e}$ and $\mathcal{S}_{\Gamma_{i}}[\psi]$ is $\mathcal{C}^{2}$ on $\Gamma_{e}$, we obtain that $\varphi \in \mathcal{C}^{2}\left(\Gamma_{e}\right)$. Likewise it follows that $\psi \in \mathcal{C}^{2}\left(\Gamma_{i}\right)$. Therefore, the function $u$ defined by $u=\mathcal{D}_{\Gamma_{e}}[\varphi]+\mathcal{S}_{\Gamma_{i}}[\psi]$ in $\Omega$ is a classical solution to (1.5), with $f=0$. Since this solution is unique, we get

$$
\mathcal{D}_{\Gamma_{e}}[\varphi]+\mathcal{S}_{\Gamma_{i}}[\psi]=0 \quad \text { in } \Omega
$$

and hence

$$
\mathcal{D}_{\Gamma_{e}}[\varphi]+\mathcal{S}_{\Gamma_{i}}[\psi]=0 \quad \text { in } U
$$

The "continuity" of the normal derivative of a double-layer potential, in combination with (2.3) now gives

$$
\begin{aligned}
\psi & =\left.\frac{\partial}{\partial \nu} \mathcal{S}_{\Gamma_{i}}[\psi]\right|_{+}-\left.\frac{\partial}{\partial \nu} \mathcal{S}_{\Gamma_{i}}[\psi]\right|_{-} \\
& =\left.\frac{\partial}{\partial \nu}\left(\mathcal{D}_{\Gamma_{e}}[\varphi]+\mathcal{S}_{\Gamma_{i}}[\psi]\right)\right|_{+}-\left.\frac{\partial}{\partial \nu}\left(\mathcal{D}_{\Gamma_{e}}[\varphi]+\mathcal{S}_{\Gamma_{i}}[\psi]\right)\right|_{-}=0 \text { on } \Gamma_{i} .
\end{aligned}
$$

In other words $\psi=0$, and $\mathcal{D}_{\Gamma_{e}}[\varphi]=0$ in $U$. It follows from (2.4) that $\left(\frac{1}{2} I+\mathcal{K}_{\Gamma_{e}}\right)[\varphi]=0$, and the invertibility of $\frac{1}{2} I+\mathcal{K}_{\Gamma_{e}}$ now insures that $\varphi=0$.

Invertibility of $A_{0}$ on $H^{1}\left(\Gamma_{e}\right) \times L^{2}\left(\Gamma_{i}\right)$ follows easily by the fact that if $(\varphi, \psi) \in L^{2}\left(\Gamma_{e}\right) \times$ $L^{2}\left(\Gamma_{i}\right)$ and $A_{0}\left(\begin{array}{l}\varphi \\ \psi\end{array}\right) \in H^{1}\left(\Gamma_{e}\right) \times L^{2}\left(\Gamma_{i}\right)$, then $\mathcal{K}_{\Gamma_{e}}[\varphi] \in H^{1}\left(\Gamma_{e}\right)$, and hence $\varphi \in H^{1}\left(\Gamma_{e}\right)$. This completes the proof.

As an immediate consequence of Lemma 3.1, we obtain the following theorem.

Theorem 3.2 The solution $u_{0}$ to (1.5) can be represented as

$$
u_{0}=\mathcal{D}_{\Gamma_{e}}\left[\varphi_{0}\right]+\mathcal{S}_{\Gamma_{i}}\left[\psi_{0}\right] \text { in } \Omega,
$$

where $\left(\varphi_{0}, \psi_{0}\right)$ is the unique solution to (3.1). 
Using the exact same techniques we may also derive a representation formula for the unique solution to the boundary value problem

$$
\begin{cases}\Delta v_{0}=0 & \text { in } \Omega, \\ -\frac{\partial v_{0}}{\partial \nu}=g & \text { on } \Gamma_{i} \\ v_{0}=0 & \text { on } \Gamma_{e},\end{cases}
$$

where $g \in L^{2}\left(\Gamma_{i}\right)$. The formula simply reads

$$
v_{0}=\mathcal{D}_{\Gamma_{e}}\left[\tilde{\varphi}_{0}\right]+\mathcal{S}_{\Gamma_{i}}\left[\tilde{\psi}_{0}\right] \text { in } \Omega,
$$

where $\left(\tilde{\varphi}_{0}, \tilde{\psi}_{0}\right)$ is the solution to

$$
A_{0}\left(\begin{array}{c}
\tilde{\varphi}_{0} \\
\tilde{\psi}_{0}
\end{array}\right)=\left(\begin{array}{l}
0 \\
g
\end{array}\right)
$$

We proceed to derive a representation formula for $u_{\epsilon}$, the solution to (1.4), valid for $\epsilon$ sufficiently small. In this case the relevant integral operator is

$$
A_{\gamma}:=\left(\begin{array}{cc}
\frac{1}{2} I+\mathcal{K}_{\Gamma_{e}} & \mathcal{S}_{\Gamma_{i}} \\
-\frac{\partial}{\partial \nu_{i}} \mathcal{D}_{\Gamma_{e}}+\gamma \mathcal{D}_{\Gamma_{e}} & -\frac{1}{2} I-\mathcal{K}_{\Gamma_{i}}^{*}+\gamma \mathcal{S}_{\Gamma_{i}}
\end{array}\right) .
$$

Observe that

$$
A_{\gamma}=A_{0}+M_{\gamma} B
$$

where $M_{\gamma}$ represents multiplication by $\gamma$, and

$$
B=\left(\begin{array}{cc}
0 & 0 \\
\mathcal{D}_{\Gamma_{e}} & \mathcal{S}_{\Gamma_{i}}
\end{array}\right) .
$$

Since

$$
\|\gamma\|_{L^{p}\left(\Gamma_{i}\right)} \leq C \epsilon^{1 / p}
$$

we have

$$
\begin{aligned}
\left\|M_{\gamma} B\left(\begin{array}{c}
\varphi \\
\psi
\end{array}\right)\right\|_{X_{p}} & \leq\|\gamma\|_{L^{p}\left(\Gamma_{i}\right)}\left\|\mathcal{D}_{\Gamma_{e}}[\varphi]+\mathcal{S}_{\Gamma_{i}}[\psi]\right\|_{L^{\infty}\left(\Gamma_{i}\right)} \\
& \leq C_{p} \epsilon^{1 / p}\left\|\left(\begin{array}{c}
\varphi \\
\psi
\end{array}\right)\right\|_{X_{p}},
\end{aligned}
$$

for any $p>1$. Here $\|(\varphi, \psi)\|_{X_{p}}:=\|\varphi\|_{L^{p}\left(\Gamma_{e}\right)}+\|\psi\|_{L^{p}\left(\Gamma_{i}\right)}$ and $C_{p}$ is a constant depending only on $p$. In other words, we have a bound for the operator norm, $\left\|M_{\gamma} B A_{0}^{-1}\right\|_{p}$, of $M_{\gamma} B A_{0}^{-1}$ on $X_{p}$ :

$$
\left\|M_{\gamma} B A_{0}^{-1}\right\|_{p} \leq C_{p} \epsilon^{1 / p}
$$


Likewise, one can show that

$$
\left\|M_{\gamma} B A_{0}^{-1}\left(\begin{array}{c}
\varphi \\
\psi
\end{array}\right)\right\|_{H^{1}\left(\Gamma_{e}\right) \times L^{2}\left(\Gamma_{i}\right)} \leq C_{2} \epsilon^{1 / 2}\left\|\left(\begin{array}{c}
\varphi \\
\psi
\end{array}\right)\right\|_{H^{1}\left(\Gamma_{e}\right) \times L^{2}\left(\Gamma_{i}\right)}
$$

Since

$$
A_{\gamma}=\left(I+M_{\gamma} B A_{0}^{-1}\right) A_{0}
$$

we arrive at the following theorem.

Theorem 3.3 There exists a positive number $\epsilon_{0}$ such that for any $0<\epsilon<\epsilon_{0} A_{\gamma}$ is invertible on $X_{p}$ and on $H^{1}\left(\Gamma_{e}\right) \times L^{2}\left(\Gamma_{i}\right)$. Moreover, for any $f \in H^{1}\left(\Gamma_{e}\right)$, the solution $u_{\epsilon}$ to (1.4) can be represented as

$$
u_{\epsilon}=\mathcal{D}_{\Gamma_{e}}\left[\varphi_{\epsilon}\right]+\mathcal{S}_{\Gamma_{i}}\left[\psi_{\epsilon}\right] \text { in } \Omega
$$

where $\left(\varphi_{\epsilon}, \psi_{\epsilon}\right) \in H^{1}\left(\Gamma_{e}\right) \times L^{2}\left(\Gamma_{i}\right)$ is the unique solution to

$$
A_{\gamma}\left(\begin{array}{l}
\varphi_{\epsilon} \\
\psi_{\epsilon}
\end{array}\right)=\left(\begin{array}{l}
f \\
0
\end{array}\right)
$$

The last result in this section gives a complete expansion for $u_{\epsilon}$ in terms of iterates easily constructed from $u_{0}$. We can only prove convergence of this expansion for $\epsilon$ sufficiently small. First note that

$$
A_{\gamma}^{-1}=A_{0}^{-1}\left(I+M_{\gamma} B A_{0}^{-1}\right)^{-1}
$$

and

$$
M_{\gamma} B\left(\begin{array}{c}
\varphi \\
\psi
\end{array}\right)=\left(\begin{array}{c}
0 \\
\gamma\left(\mathcal{D}_{\Gamma_{e}}[\varphi]+\mathcal{S}_{\Gamma_{i}}[\psi]\right)
\end{array}\right)
$$

By expanding (3.8) in a Neumann series, we get

$$
A_{\gamma}^{-1}=A_{0}^{-1}+\sum_{n=1}^{+\infty}(-1)^{n} A_{0}^{-1}\left(M_{\gamma} B A_{0}^{-1}\right)^{n}
$$

Now let

$$
\left(\begin{array}{l}
\varphi_{n} \\
\psi_{n}
\end{array}\right):=A_{0}^{-1}\left(M_{\gamma} B A_{0}^{-1}\right)^{n}\left(\begin{array}{l}
f \\
0
\end{array}\right), \quad n=0,1,2, \ldots
$$

then

$$
\left(\begin{array}{l}
\varphi_{\epsilon} \\
\psi_{\epsilon}
\end{array}\right)=A_{\gamma}^{-1}\left(\begin{array}{l}
f \\
0
\end{array}\right)=\sum_{n=0}^{+\infty}(-1)^{n}\left(\begin{array}{l}
\varphi_{n} \\
\psi_{n}
\end{array}\right) .
$$

Moreover, the following recursive relation holds:

$$
\left(\begin{array}{l}
\varphi_{n+1} \\
\psi_{n+1}
\end{array}\right)=A_{0}^{-1} M_{\gamma} B\left(\begin{array}{l}
\varphi_{n} \\
\psi_{n}
\end{array}\right), \quad n=0,1,2, \ldots .
$$

It then follows from (3.6) and (3.10) that

$$
\left\|\varphi_{n}\right\|_{H^{1}\left(\Gamma_{e}\right)}+\left\|\psi_{n}\right\|_{L^{2}\left(\Gamma_{i}\right)} \leq C_{0} C^{n} \epsilon^{n / 2}\|f\|_{H^{1}\left(\Gamma_{e}\right)}, \quad n=0,1,2, \ldots,
$$

for some constant $C$ independent of $\epsilon$. Here $C_{0}$ is a bound for $A_{0}^{-1}$. 
If we define

$$
u_{n}:=\mathcal{D}_{\Gamma_{e}}\left[\varphi_{n}\right]+\mathcal{S}_{\Gamma_{i}}\left[\psi_{n}\right] \quad \text { in } \Omega, \quad n=0,1,2, \ldots,
$$

which as far as $u_{0}$ is concerned is consistent with being the solution to (1.5), then the identity (3.11) immediately yields

$$
u_{\epsilon}=\sum_{n=0}^{+\infty}(-1)^{n} u_{n} \quad \text { in } \Omega
$$

It is not difficult to see that this series converges in $H^{3 / 2}(\Omega)$ (for $\epsilon$ sufficiently small). In fact, thanks to elliptic estimates, we have

$$
\begin{aligned}
\left\|u_{n}\right\|_{H^{3 / 2}(\Omega)} & \leq C^{\prime}\left(\left\|\left.\mathcal{D}_{\Gamma_{e}}\left[\varphi_{n}\right]\right|_{-}\right\|_{H^{1}\left(\Gamma_{e}\right)}+\left\|\mathcal{S}_{\Gamma_{i}}\left[\psi_{n}\right]\right\|_{H^{1}\left(\Gamma_{i}\right)}\right) \\
& \leq C^{\prime}\left(\left\|\left(\frac{1}{2} I+\mathcal{K}_{\Gamma_{e}}\right)\left[\varphi_{n}\right]\right\|_{H^{1}\left(\Gamma_{e}\right)}+\left\|\mathcal{S}_{\Gamma_{i}}\left[\psi_{n}\right]\right\|_{H^{1}\left(\Gamma_{i}\right)}\right) \\
& \leq C_{1}\left(\left\|\varphi_{n}\right\|_{H^{1}\left(\Gamma_{e}\right)}+\left\|\psi_{n}\right\|_{L^{2}\left(\Gamma_{i}\right)}\right)
\end{aligned}
$$

for some constant $C^{\prime}$ and $C_{1}$, and hence, by (3.13),

$$
\left\|u_{n}\right\|_{H^{3 / 2}(\Omega)} \leq C_{0} C_{1} C^{n} \epsilon^{n / 2}\|f\|_{H^{1}\left(\Gamma_{e}\right)} .
$$

Thus the series for $u_{\epsilon}$ converges strongly in $H^{3 / 2}(\Omega)$ as long as $\epsilon$ is sufficiently small.

By (3.9) and (3.12),

$$
\left(\begin{array}{l}
\varphi_{n} \\
\psi_{n}
\end{array}\right)=A_{0}^{-1}\left(\begin{array}{c}
0 \\
\gamma\left(\mathcal{D}_{\Gamma_{e}}\left[\varphi_{n-1}\right]+\mathcal{S}_{\Gamma_{i}}\left[\psi_{n-1}\right]\right)
\end{array}\right)=A_{0}^{-1}\left(\begin{array}{c}
0 \\
\left.\gamma u_{n-1}\right|_{\Gamma_{i}}
\end{array}\right), \quad n \geq 1
$$

and so $u_{n}, n \geq 1$, is the solution to

$$
\begin{cases}\Delta u_{n}=0 & \text { in } \Omega, \\ -\frac{\partial u_{n}}{\partial \nu}=\gamma u_{n-1} & \text { on } \Gamma_{i}, \\ u_{n}=0 & \text { on } \Gamma_{e} .\end{cases}
$$

Here we have used the observation immediately following Theorem 3.2. In summary we have proven:

Theorem 3.4 There exists a positive number $\epsilon_{0}$ such that, for any $0<\epsilon<\epsilon_{0}$, and any $f \in H^{1}\left(\Gamma_{e}\right)$, the solution $u_{\epsilon}$ to (1.4) has the expansion

$$
u_{\epsilon}=\sum_{n=0}^{+\infty}(-1)^{n} u_{n} \quad \text { in } H^{3 / 2}(\Omega)
$$

where $u_{0}$ is the solution to (1.5) and $u_{n}, n \geq 1$, are the solutions to (3.16). 


\section{Asymptotic Expansion}

We now derive a formula for the principal term of $\frac{\partial}{\partial \nu}\left(u_{\epsilon}-u_{0}\right)$ (on $\Gamma_{e}$ ) as $\epsilon \rightarrow 0$. This formula forms the basis for our corrosion detection algorithm. In order to derive our formula we need an elliptic regularity estimate for the solution to the boundary value problem

$$
\left\{\begin{array}{ll}
\Delta v=0 & \text { in } \Omega \\
-\frac{\partial v}{\partial \nu}=\gamma g & \text { on } \Gamma_{i} \\
v=0 & \text { on } \Gamma_{e}
\end{array} .\right.
$$

In particular we need that

$$
\left\|\frac{\partial v}{\partial \nu}\right\|_{\mathcal{C}^{1}\left(\Gamma_{e}\right)} \leq C_{p}\|\gamma g\|_{L^{p}\left(\Gamma_{i}\right)}
$$

for any $p>1$. This regularity estimate is a consequence of well-known Schauder estimates, and could just be stated without proof - however, for completeness we show how it also immediately follows from our integral representation of the solution. We have seen that the solution $v$ to $(4.1)$ can be represented as

$$
v=\mathcal{D}_{\Gamma_{e}}[\varphi]+\mathcal{S}_{\Gamma_{i}}[\psi] \text { in } \Omega,
$$

where $(\varphi, \psi)$ is the solution to

$$
A_{0}\left(\begin{array}{c}
\varphi \\
\psi
\end{array}\right)=\left(\begin{array}{c}
0 \\
\gamma g
\end{array}\right) \text {, }
$$

i.e. ,

$$
\begin{cases}\left(\frac{1}{2} I+\mathcal{K}_{\Gamma_{e}}\right)[\varphi]+\mathcal{S}_{\Gamma_{i}}[\psi]=0 & \text { on } \Gamma_{e}, \\ -\frac{\partial}{\partial \nu} \mathcal{D}_{\Gamma_{e}}[\varphi]-\left(\frac{1}{2} I+\mathcal{K}_{\Gamma_{i}}^{*}\right)[\psi]=\gamma g & \text { on } \Gamma_{i} .\end{cases}
$$

Since $A_{0}$ is invertible on $X_{p}$, we have

$$
\|\psi\|_{L^{p}\left(\Gamma_{i}\right)} \leq C_{p}\|\gamma g\|_{L^{p}\left(\Gamma_{i}\right)} .
$$

Let $2 \delta=\operatorname{dist}\left(\Gamma_{i}, \Gamma_{e}\right)$, and define $\Omega_{\delta}:=\left\{x \in \Omega: \operatorname{dist}\left(x, \Gamma_{e}\right) \leq \delta\right\}$. Then

$$
\left\|\mathcal{S}_{\Gamma_{i}}[\psi]\right\|_{\mathcal{C}^{2, \alpha}\left(\Omega_{\delta}\right)} \leq C\|\psi\|_{L^{p}\left(\Gamma_{i}\right)} \leq C_{p}\|\gamma g\|_{L^{p}\left(\Gamma_{i}\right)},
$$

where constants depend on $\delta$. It now follows from the first equation in (4.3) and Lemma 2.1 that

$$
\begin{aligned}
\|\varphi\|_{\mathcal{C}^{2, \alpha}\left(\Gamma_{e}\right)} & =\left\|\left(\frac{1}{2} I+\mathcal{K}_{\Gamma_{e}}\right)^{-1}\left[\left.\left(\mathcal{S}_{\Gamma_{i}}[\psi]\right)\right|_{\Gamma_{i}}\right]\right\|_{\mathcal{C}^{2, \alpha}\left(\Gamma_{e}\right)} \\
& \leq C\left\|\mathcal{S}_{\Gamma_{i}}[\psi]\right\|_{\mathcal{C}^{2, \alpha}\left(\Gamma_{e}\right)} \\
& \leq C_{p}\|\gamma g\|_{L^{p}\left(\Gamma_{i}\right)} .
\end{aligned}
$$


Note that if $\varphi \in \mathcal{C}^{2, \alpha}\left(\Gamma_{e}\right)$, then $\mathcal{D}_{\Gamma_{e}}[\varphi] \in \mathcal{C}^{2, \alpha}(U)$. In fact, if $\varphi \in \mathcal{C}^{2, \alpha}\left(\Gamma_{e}\right)$, then $\mathcal{K}_{\Gamma_{e}}[\varphi] \in$ $\mathcal{C}^{2, \alpha}\left(\Gamma_{e}\right)$. Since $u:=\mathcal{D}_{\Gamma_{e}}[\varphi]$ is the unique solution to the Dirichlet problem $\Delta u=0$ in $U$ and $\left.u\right|_{\Gamma_{e}}=\frac{1}{2} \varphi+\mathcal{K}_{\Gamma_{e}}[\varphi]$, we have $\mathcal{D}_{\Gamma_{e}}[\varphi] \in \mathcal{C}^{2, \alpha}(U)$. Therefore, we have in particular

$$
\left\|\frac{\partial v}{\partial \nu}\right\|_{\mathcal{C}^{1}\left(\Gamma_{e}\right)} \leq\left\|\mathcal{D}_{\Gamma_{e}}[\varphi]+\mathcal{S}_{\Gamma_{i}}[\psi]\right\|_{\mathcal{C}^{2}\left(\Omega_{\delta}\right)} \leq C_{p}\|\gamma g\|_{L^{p}\left(\Gamma_{i}\right)}
$$

which is exactly the estimate (4.2). By a combination of (4.2) with the estimate

$$
\|\gamma g\|_{L^{p}\left(\Gamma_{i}\right)} \leq C \epsilon^{1 / p}\|g\|_{L^{\infty}\left(\Gamma_{i}\right)},
$$

we obtain the following lemma.

Lemma 4.1 Let $v$ be the solution to (4.1). Then, for each $p>1$, there is a constant $C_{p}$, independent of $g$, such that

$$
\left\|\frac{\partial v}{\partial \nu}\right\|_{\mathcal{C}^{1}\left(\Gamma_{e}\right)} \leq C_{p} \epsilon^{1 / p}\|g\|_{L^{\infty}\left(\Gamma_{i}\right)} .
$$

Based on (3.16), (3.17), and (4.6)

$$
\sum_{n=k}^{+\infty}\left\|\frac{\partial u_{n}}{\partial \nu}\right\|_{\mathcal{C}^{1}\left(\Gamma_{e}\right)} \leq C_{p} \epsilon^{1 / p} \sum_{n=k}^{+\infty}\left\|u_{n-1}\right\|_{L^{\infty}\left(\Gamma_{i}\right)},
$$

for any $k \geq 1$. From (3.14) we have

$$
\left\|u_{n-1}\right\|_{L^{\infty}\left(\Gamma_{i}\right)}=\left\|\mathcal{D}_{\Gamma_{e}}\left[\varphi_{n-1}\right]+\mathcal{S}_{\Gamma_{i}}\left[\psi_{n-1}\right]\right\|_{L^{\infty}\left(\Gamma_{i}\right)} \leq C_{p}\left\|\left(\begin{array}{c}
\varphi_{n-1} \\
\psi_{n-1}
\end{array}\right)\right\|_{X_{p}}
$$

which in combination with (3.5) and (3.10) yields

$$
\left\|u_{n-1}\right\|_{L^{\infty}\left(\Gamma_{i}\right)} \leq C_{p}\left(C \epsilon^{1 / p}\right)^{n-1}\|f\|_{L^{p}\left(\Gamma_{e}\right)} .
$$

Insertion into (4.7) now gives

$$
\sum_{n=k}^{+\infty}\left\|\frac{\partial u_{n}}{\partial \nu}\right\|_{\mathcal{C}^{1}\left(\Gamma_{e}\right)} \leq C_{p} \epsilon^{1 / p} \sum_{n=k}^{+\infty}\left(C \epsilon^{1 / p}\right)^{n-1}\|f\|_{L^{p}\left(\Gamma_{e}\right)} \leq C_{k, p} \frac{\epsilon^{k / p}}{1-C \epsilon^{1 / p}}\|f\|_{L^{p}\left(\Gamma_{e}\right)}
$$

for any $k \geq 1$, provided $C \epsilon^{1 / p}<1$. The preceeding analysis immediately leads to the following theorem.

Theorem 4.2 For all $p>1$, there is a constant $C_{p}$ such that

$$
\left\|\frac{\partial u_{\epsilon}}{\partial \nu}-\frac{\partial u_{0}}{\partial \nu}\right\|_{\mathcal{C}^{1}\left(\Gamma_{e}\right)} \leq C_{p} \epsilon^{1 / p}\|f\|_{L^{p}\left(\Gamma_{e}\right)}
$$

We now derive the desired formula for the principal term of $\frac{\partial u_{\epsilon}}{\partial \nu}-\frac{\partial u_{0}}{\partial \nu}$. Using the notation from before we easily obtain that

$$
\frac{\partial u_{\epsilon}}{\partial \nu}(x)-\frac{\partial u_{0}}{\partial \nu}(x)=-\frac{\partial u_{1}}{\partial \nu}(x)+O\left(\epsilon^{2 / p}\right),
$$


uniformly on $\Gamma_{e}$. We thus need to derive a formula for the principal term of $\frac{\partial u_{1}}{\partial \nu}$. For that purpose, let $G(x, y)$ denote the Green's function for the problem (3.3), i.e., for each $x \in \Omega$, $G(x, y)$ is the solution to

$$
\begin{cases}\Delta_{y} G(x, y)=-\delta_{x}(y) & \text { in } \Omega, \\ \frac{\partial}{\partial \nu_{y}} G(x, y)=0, & y \in \Gamma_{i}, \\ G(x, y)=0, & y \in \Gamma_{e} .\end{cases}
$$

By continuity $G(\cdot, \cdot)$ may be extended to all of $\bar{\Omega} \times \bar{\Omega} \backslash\{(x, y): x=y\}$, and it satisfies $G(x, y)=G(y, x)$. In terms of $G$ the solution $v$ to (4.1) can be written

$$
v(x)=\int_{\Gamma_{i}} G(x, y) \gamma(y) g(y) d \sigma_{y}, \quad x \in \Omega .
$$

Remember: $\nu$ is the inward normal to $\Omega$ on $\Gamma_{i}$. With $g=u_{0}$ this yields

$$
u_{1}(x)=\int_{\Gamma_{i}} G(x, y) \gamma(y) u_{0}(y) d \sigma_{y}, \quad x \in \Omega,
$$

and, by taking the normal derivative on $\Gamma_{e}$ we now obtain

$$
\frac{\partial u_{1}}{\partial \nu}(x)=\int_{\Gamma_{i}} \frac{\partial}{\partial \nu_{x}} G(x, y) \gamma(y) u_{0}(y) d \sigma_{y}, \quad x \in \Gamma_{e} .
$$

Note that $\frac{\partial}{\partial \nu_{x}} G(x, \cdot)$ is a $\mathcal{C}^{2}$ function on $\Gamma_{i}$ for any $x \in \Gamma_{e}$ and that $u_{0}$ is also $\mathcal{C}^{2}$ on $\Gamma_{i}$ since $\Gamma_{i}$ is away from $\Gamma_{e}$. Thus we have

$$
\frac{\partial}{\partial \nu_{x}} G(x, y) \gamma(y) u_{0}(y)=\sum_{s=1}^{m}\left[\frac{\partial}{\partial \nu_{x}} G\left(x, z_{s}\right) u_{0}\left(z_{s}\right)+O(\epsilon)\right] \gamma_{s}(y) \chi_{s}(y)
$$

where $\chi_{s}$ is the characteristic function of $I_{s}$. We thus finally get

$$
\frac{\partial u_{1}}{\partial \nu}(x)=\sum_{s=1}^{m} \frac{\partial}{\partial \nu_{x}} G\left(x, z_{s}\right) u_{0}\left(z_{s}\right) \int_{I_{s}} \gamma_{s} d \sigma+O\left(\epsilon^{2}\right)
$$

Define

$$
\langle\gamma\rangle_{s}:=\int_{I_{s}} \gamma_{s} d \sigma
$$

Then we have arrived at the following formula for the principal term of $\frac{\partial}{\partial \nu} u_{\epsilon}-\frac{\partial}{\partial \nu} u_{0}$.

Theorem 4.3 For $x \in \Gamma_{e}$ :

$$
\frac{\partial u_{\epsilon}}{\partial \nu}(x)-\frac{\partial u_{0}}{\partial \nu}(x)=-\sum_{s=1}^{m}\langle\gamma\rangle_{s} u_{0}\left(z_{s}\right) \frac{\partial}{\partial \nu_{x}} G\left(x, z_{s}\right)+O\left(\epsilon^{1+\beta}\right)
$$

for any $0<\beta<1$. 


\section{MUSIC type algorithm for reconstruction}

We now apply the asymptotic formula (4.10) to design a MUSIC (MUltiple SIgnal Classification) algorithm for locating small internal corrosive parts from boundary measurements. MUSIC is generally used in signal processing problems as a method for estimating the individual frequencies from multiple-harmonic signals [20]. In the present context the MUSIC algorithm uses information about the spectral structure of the (exterior Dirichletto-Neumann) boundary map for the Laplace operator with the "internal" corrosion (Robin) boundary condition. The eigenvectors corresponding to the most significant eigenvalues span a "signal subspace" in the sense that they contain nearly all the information about the corrosive parts that can be extracted from the boundary map. The remaining eigenvectors span a sort of "noise subspace". In order to be of any practical interest our algorithm uses spectral information about a particular discrete version of the boundary map.

It is worth mentioning that the present algorithm is also related to the linear sampling method of Colton and Kirsch [9]. We refer to Cheney [7], and Kirsch [18], for detailed discussions of the connection between MUSIC-type algorithms and the linear sampling method.

Define the (exterior Dirichlet-to-Neumann) map $\Lambda_{\gamma}$ from $H^{1 / 2}\left(\Gamma_{e}\right)$ into $H^{-1 / 2}\left(\Gamma_{e}\right)$ by

$$
\Lambda_{\gamma}(f)=\left.\frac{\partial u_{\epsilon}}{\partial \nu}\right|_{\Gamma_{e}}
$$

where $u_{\epsilon}$ is the solution to (1.4). Let $\Lambda_{0}$ be the exterior Dirichlet-to-Neumann map for the case when no corrosion is present. We proceed to show how partial spectral information about $\Lambda_{\gamma}-\Lambda_{0}$ may effectively be used to determine the corroded internal boundary parts. The estimate (4.8) immediately gives that

$$
\left\|\left(\Lambda_{\gamma}-\Lambda_{0}\right)(f)\right\|_{\mathcal{C}^{1}\left(\Gamma_{e}\right)} \leq C_{p} \epsilon^{1 / p}\|f\|_{L^{2}\left(\Gamma_{e}\right)},
$$

for any $1<p \leq 2$. In particular this shows that the operator $\Lambda_{\gamma}-\Lambda_{0}$, originally defined on $H^{1 / 2}\left(\Gamma_{e}\right)$, can be extended as an operator on all of $L^{2}\left(\Gamma_{e}\right)$. This operator is compact when regarded as an operator from $L^{2}\left(\Gamma_{e}\right)$ into itself. We also note that for $g \in H^{1 / 2}\left(\Gamma_{e}\right)$

$$
\int_{\Gamma_{e}}\left(\Lambda_{\gamma}-\Lambda_{0}\right)(f) g d \sigma=\int_{\Omega}\left[\nabla u_{\epsilon} \cdot \nabla v_{\epsilon}-\nabla u_{0} \cdot \nabla v_{0}\right] d x+\int_{\Gamma_{i}} \gamma u_{\epsilon} v_{\epsilon} d \sigma
$$

where $v_{\epsilon}$ and $v_{0}$ are solutions to (1.4) and (1.5) with $f$ replaced by $g$, respectively. Note that

$$
\int_{\Omega} \nabla u_{\epsilon} \cdot \nabla u_{0} d x=\int_{\Gamma_{e}} f \frac{\partial u_{0}}{\partial \nu} d \sigma=\int_{\Omega} \nabla u_{0} \cdot \nabla u_{0} d x .
$$

Thus we obtain by substituting $f$ in place of $g$ in (5.3)

$$
\int_{\Gamma_{e}}\left(\Lambda_{\gamma}-\Lambda_{0}\right) f f d \sigma=\int_{\Omega}\left|\nabla\left(u_{\epsilon}-u_{0}\right)\right|^{2} d x+\int_{\Gamma_{i}} \gamma\left|u_{\epsilon}\right|^{2} d \sigma .
$$

In summary we have established the following lemma.

Lemma $5.1 \Lambda_{\gamma}-\Lambda_{0}$ is self-adjoint, positive, and compact on $L^{2}\left(\Gamma_{e}\right)$.

The identity (4.10) shows that

$$
\left(\Lambda_{\gamma}-\Lambda_{0}\right)(f)(x)=-\sum_{s=1}^{m}\langle\gamma\rangle_{s} u_{0}\left(z_{s}\right) \frac{\partial}{\partial \nu_{x}} G\left(x, z_{s}\right)+O\left(\epsilon^{1+\beta}\right), \quad x \in \Gamma_{e},
$$


for any $0<\beta<1$. It is not difficult to see that the remainder term $O\left(\epsilon^{1+\beta}\right)$ is actually bounded by $C_{\beta} \epsilon^{1+\beta}\|f\|_{L^{2}\left(\Gamma_{e}\right)}$ with $C_{\beta}$ independent of $f$. Define the operator $T$ on $H^{1 / 2}\left(\Gamma_{e}\right)$ by

$$
(T f)(x)=-\sum_{s=1}^{m}\langle\gamma\rangle_{s} u_{0}\left(z_{s}\right) \frac{\partial}{\partial \nu_{x}} G\left(x, z_{s}\right), \quad x \in \Gamma_{e} .
$$

Since $u_{0}$ depends linearly on $f, T$ is a linear operator and can be extended as a bounded operator on $L^{2}\left(\Gamma_{e}\right)$. Furthermore the estimate we have just verified may easily be sharpened to show that

$$
\Lambda_{\gamma}-\Lambda_{0}=T+O\left(\epsilon^{1+\beta}\right)
$$

in the operator norm on $L^{2}\left(\Gamma_{e}\right)$.

Lemma 5.2 The operator $T$ is a compact, self-adjoint, positive semi-definite operator on $L^{2}\left(\Gamma_{e}\right)$.

If $\langle\gamma\rangle_{s}=c_{s} \epsilon$ for some fixed constants $c_{s}$, then this follows immediately by multiplication of (5.4) by $\epsilon^{-1}$, consideration of the $\epsilon \rightarrow 0$ limit, and use of Lemma 5.1. Here it is essential that the remainder term is bounded by $C_{\beta} \epsilon^{1+\beta}\|f\|_{L^{2}\left(\Gamma_{e}\right)}$, i.e., that we have norm convergence. More generally the lemma can also be verified by noting that $T$ is a finite-dimensional operator, and that $u_{0}$ is given by

$$
u_{0}(y)=-\int_{\Gamma_{e}} \frac{\partial}{\partial \nu_{x}} G(x, y) f(x) d \sigma(x), \quad y \in \Omega
$$

so that

$$
\int_{\Gamma_{e}} T(f) h=\sum_{s=1}^{m}\langle\gamma\rangle_{s} u_{0}\left(z_{s}\right) v_{0}\left(z_{s}\right)
$$

where $v_{0}$ is the solution to (1.5), with $f$ replaced by $h$.

We introduce the linear operator $\mathcal{G}: L^{2}\left(\Gamma_{e}\right) \rightarrow \mathbb{R}^{m}$

$$
\mathcal{G} f=\left(u_{0}\left(z_{1}\right), \ldots, u_{0}\left(z_{m}\right)\right),
$$

where $u_{0}$, as before, is the solution to (1.5). If $\mathbb{R}^{m}$ is endowed with the standard Euclidean inner product then

$$
\langle\mathcal{G} f, a\rangle=\sum_{s=1}^{m} a_{s} u_{0}\left(z_{s}\right)=-\int_{\Gamma_{e}}\left(\sum_{s=1}^{m} a_{s} \frac{\partial}{\partial \nu_{x}} G\left(x, z_{s}\right)\right) f(x) d \sigma(x),
$$

for arbitrary $a=\left(a_{1}, \ldots, a_{m}\right) \in \mathbb{R}^{m}$. Therefore, the adjoint operator $\mathcal{G}^{*}: \mathbb{R}^{m} \rightarrow L^{2}\left(\Gamma_{e}\right)$ is given by

$$
\mathcal{G}^{*} a=-\sum_{s=1}^{m} a_{s} \frac{\partial}{\partial \nu_{x}} G\left(\cdot, z_{s}\right) .
$$

Exactly as in Brühl et al. [4] there is a simple factorization of the operator $T$, and a corresponding characterization of its range.

\section{Lemma 5.3}


(i) $\mathcal{G}^{*}$ is injective;

(ii) $\mathcal{G}$ is surjective;

(iii) $T=\mathcal{G}^{*} \mathcal{M G}$, where

$$
\mathcal{M} a=\left(\langle\gamma\rangle_{1} a_{1}, \ldots,\langle\gamma\rangle_{m} a_{m}\right), \quad a=\left(a_{1}, \ldots, a_{m}\right) \in \mathbb{R}^{m}
$$

(iv) Range $(T)=\operatorname{span}\left\{\frac{\partial}{\partial \nu_{x}} G\left(x, z_{s}\right) ; s=1, \ldots, m\right\}$.

Proof. Suppose $\mathcal{G}^{*} a=0$, and let $w$ denote the function $w(x)=\sum_{s=1}^{m} a_{s} G\left(x, z_{s}\right)$. Then $w=\frac{\partial w}{\partial \nu}=0$ on $\Gamma_{e}$, and therefore by unique continuation $w(x)=\sum_{s=1}^{m} a_{s} G\left(x, z_{s}\right)=0$ in all of $\Omega$. It immediately follows that $a_{s}=0$ for $s=1, \ldots, m$. Assertion (ii) follows from (i) and the well-known relation between the range and the null space of adjoint finite-dimensional operators: Range $(\mathcal{G})=\operatorname{Ker}\left(\mathcal{G}^{*}\right)^{\perp}$. Using (5.5), (5.8), and (5.9), it is easy to see that (iii) holds. Now, according to (iii) we have $\operatorname{Range}(T)=\operatorname{Range}\left(\mathcal{G}^{*} \mathcal{M G}\right)=\operatorname{Range}\left(\mathcal{G}^{*}\right)$, since $\mathcal{M}$ and $\mathcal{G}$ are surjective. This yields (iv), and the proof is complete.

The following result forms the basis for our algorithm to identify the midpoint locations, $z_{s}$, of the small corroded boundary parts.

Theorem $5.4 A$ point $z \in \Gamma_{i}$ belongs to the set $\left\{z_{s}: s=1, \ldots, m\right\}$ if and only if

$$
\left.\frac{\partial}{\partial \nu_{x}} G(\cdot, z)\right|_{\Gamma_{e}} \in \text { Range }(T) \text {. }
$$

Proof. Assume $\left.\frac{\partial}{\partial \nu_{x}} G(\cdot, z)\right|_{\Gamma_{e}} \in$ Range $(T)$. As a consequence of (iv) of Lemma 5.3 there exist coefficients $a_{s}, 1 \leq s \leq m$ such that

$$
\frac{\partial}{\partial \nu_{x}} G(x, z)=\sum_{s=1}^{m} a_{s} \frac{\partial}{\partial \nu_{x}} G\left(x, z_{s}\right) \quad, \quad \text { for } x \in \Gamma_{e} .
$$

Since $G(x, z)=0$ for any $x \in \Gamma_{e}$ and $z \in \Gamma_{i}$, we also have

$$
G(x, z)=\sum_{s=1}^{m} a_{s} G\left(x, z_{s}\right) \quad, \quad \text { for } x \in \Gamma_{e}
$$

and by unique continuation it follows that $G(x, z)=\sum_{s=1}^{m} a_{s} G\left(x, z_{s}\right)$ for all $x \in \Omega$. Due to the singularity of $G(x, z)$ at $x=z$ this is only possible if $z \in\left\{z_{s}: s=1, \ldots, m\right\}$, and so we have established the sufficiency of the condition (5.10). The necessity follows immediately from (iv) of Lemma 5.3.

The finite-dimensional, self-adjoint operator $T$ has a spectral decomposition

$$
T=\sum_{p=1}^{m} \lambda_{p} v_{p} \otimes v_{p}, \quad\left\|v_{p}\right\|_{L^{2}\left(\Gamma_{e}\right)}=1,
$$

where $\lambda_{p}, 1 \leq p \leq m$, are the (positive) eigenvalues of $T$, and $v_{p}$ is the corresponding eigenfunction. Note that the rank of $T, m$, is exactly the number of corroded parts. We 
assume that $\lambda_{1} \geq \lambda_{2} \geq \ldots \geq \lambda_{m}>0$. Let $P_{k}: L_{0}^{2}\left(\Gamma_{e}\right) \rightarrow$ span $\left\{v_{1}, \ldots, v_{k}\right\}, k=1, \ldots, m$, be the orthogonal projector $P_{k}=\sum_{p=1}^{k} v_{p} \otimes v_{p}$. It then follows from Theorem 5.4 that

$$
z \in\left\{z_{s}: s=1, \ldots, m\right\} \quad \text { iff } \quad\left(I-P_{m}\right)\left(\left.\frac{\partial}{\partial \nu_{x}} G(\cdot, z)\right|_{\Gamma_{e}}\right)=0
$$

or equivalently,

$$
z \in\left\{z_{s}: s=1, \ldots, m\right\} \quad \text { iff } \quad \cot \theta(z)=+\infty,
$$

where the angle $\theta(z) \in[0, \pi / 2)$ is defined by

$$
\cot \theta(z)=\frac{\left\|P_{m}\left(\left.\frac{\partial}{\partial \nu_{x}} G(\cdot, z)\right|_{\Gamma_{e}}\right)\right\|_{L^{2}\left(\Gamma_{e}\right)}}{\left\|\left(I-P_{m}\right)\left(\left.\frac{\partial}{\partial \nu_{x}} G(\cdot, z)\right|_{\Gamma_{e}}\right)\right\|_{L^{2}\left(\Gamma_{e}\right)}} .
$$

Since $\Lambda_{\gamma}-\Lambda_{0}$ is a self-adjoint, positive, and compact operator on $L^{2}\left(\Gamma_{e}\right)$, it admits a spectral decomposition

$$
\Lambda_{\gamma}-\Lambda_{0}=\sum_{p=1}^{+\infty} \lambda_{p}^{\epsilon} v_{p}^{\epsilon} \otimes v_{p}^{\epsilon}, \quad\left\|v_{p}^{\epsilon}\right\|_{L^{2}\left(\Gamma_{e}\right)}=1
$$

with $\lambda_{1}^{\epsilon} \geq \lambda_{2}^{\epsilon} \geq \ldots \geq \lambda_{m}^{\epsilon} \geq \ldots>0$. Let $P_{k}^{\epsilon}: L^{2}\left(\Gamma_{e}\right) \rightarrow \operatorname{span}\left\{v_{1}^{\epsilon}, \ldots, v_{k}^{\epsilon}\right\}, k=1,2, \ldots$, be the orthogonal projector $P_{k}^{\epsilon}=\sum_{p=1}^{k} v_{p}^{\epsilon} \otimes v_{p}^{\epsilon}$.

The data for our identification algorithm consist of (an appropriate approximation to) the spectral decomposition of $\Lambda_{\gamma}-\Lambda_{0}$. Motivated by (5.10), we seek to find those points $z \in \Gamma_{i}$ such that

$$
\left.\frac{\partial}{\partial \nu_{x}} G(\cdot, z)\right|_{\Gamma_{e}} \in \text { Range }\left(\Lambda_{\gamma}-\Lambda_{0}\right) .
$$

This condition is fulfilled approximately by $z \in \Gamma_{i}$ if the following quantity is sufficiently large:

$$
\cot \theta_{k}^{\epsilon}(z):=\frac{\left\|P_{k}^{\epsilon}\left(\left.\frac{\partial}{\partial \nu_{x}} G(\cdot, z)\right|_{\Gamma_{e}}\right)\right\|_{L^{2}\left(\Gamma_{e}\right)}}{\left\|\left(I-P_{k}^{\epsilon}\right)\left(\left.\frac{\partial}{\partial \nu_{x}} G(\cdot, z)\right|_{\Gamma_{e}}\right)\right\|_{L^{2}\left(\Gamma_{e}\right)}} .
$$

Since we don't a priory know $m$, we plot the angles $\theta_{k}^{\epsilon}(z)$ (for $k=1,2, \ldots$ ) as a function of $z$. When the plots "stabilize", i.e., don't significantly change by increasing $k$, we have a good estimate for $m$, and we interpret locations where small angles are attained as good candidates for the $z_{s}$.

Once the locations $z_{s}$ are approximately found, we can also estimate the integrated corrosion coefficients $\langle\gamma\rangle_{s}$. Our procedure for doing this is the following. Define $w_{s}, s=$ $1, \ldots, m$, to be the solution to

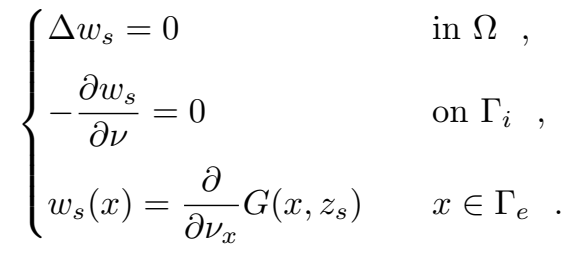


It then follows from (5.4) and (5.15) that

$$
\begin{aligned}
-\sum_{s=1}^{m}\langle\gamma\rangle_{s} w_{s^{\prime}}\left(z_{s}\right) \frac{\partial}{\partial \nu_{x}} G\left(\cdot, z_{s}\right) & \approx\left(\Lambda_{\gamma}-\Lambda_{0}\right)\left(\frac{\partial}{\partial \nu_{x}} G\left(\cdot, z_{s^{\prime}}\right)\right) \\
& \approx \sum_{p=1}^{m} \lambda_{p}^{\epsilon}\left\langle v_{p}^{\epsilon}, \frac{\partial}{\partial \nu_{x}} G\left(\cdot, z_{s^{\prime}}\right)\right\rangle v_{p}^{\epsilon} .
\end{aligned}
$$

By integrating both sides of the above formula against $v_{s^{\prime}}$, we obtain

$$
-\sum_{s=1}^{m} w_{s^{\prime}}\left(z_{s}\right)\left\langle v_{s^{\prime}}^{\epsilon}, \frac{\partial}{\partial \nu_{x}} G\left(\cdot, z_{s}\right)\right\rangle\langle\gamma\rangle_{s} \approx \lambda_{s^{\prime}}^{\epsilon}\left\langle v_{s^{\prime}}^{\epsilon}, \frac{\partial}{\partial \nu_{x}} G\left(\cdot, z_{s^{\prime}}\right)\right\rangle,
$$

for $s^{\prime}=1, \ldots, m$. Therefore the values of $\langle\gamma\rangle_{s}, s=1, \ldots, m$, can be calculated by solving a linear system. For the simplest case, $m=1$, the formula reads

$$
\langle\gamma\rangle_{1} \approx-\frac{\lambda_{1}^{\epsilon}}{w_{1}\left(z_{1}\right)}
$$

\section{$5.1 \quad$ Numerical Results}

In this section we present some numerical experiments with the MUSIC-type algorithm we have designed to find the internally corroded parts, $I_{s} \subset \mathbb{R}^{2}, s=1,2, \cdots, m$. The domain $\Omega \subset \mathbb{R}^{2}$ is taken to be an annulus, i.e., $\Omega=U \backslash \bar{D}$, where $U$ and $D$ are disks centered at $(0,0)$ with radii $r_{e}$ and $r_{i}$, respectively. The measurements used for the identification represent a discrete analogue of the Dirichlet-to-Neumann map $\Lambda_{\gamma}-\Lambda_{0}$ on $\Gamma_{e}$. In order to find a good approximation from the "identification point of view" the following observation will be useful.

Lemma 5.5 The functions $\frac{\partial G}{\partial \nu_{x}}(\cdot, y), y \in \Gamma_{i}$ span a dense subspace of $L^{2}\left(\Gamma_{e}\right)$.

Proof. The solution to the boundary value problem (1.5), for a given $f \in L^{2}\left(\Gamma_{e}\right)$, is given by

$$
u_{0}(y)=-\int_{\Gamma_{e}} \frac{\partial}{\partial \nu_{x}} G(x, y) f(x) d \sigma(x) .
$$

If $f \in L^{2}\left(\Gamma_{e}\right)$ were orthogonal to $\left\{\frac{\partial G}{\partial \nu_{x}}(\cdot, y): y \in \Gamma_{i}\right\}$ it thus follows that

$$
\frac{\partial u_{0}}{\partial \nu}=u_{0}=0 \quad \text { on } \Gamma_{i}
$$

By unique continuation this implies that $u_{0}=0$ in all of $\Omega$, and so consequently $f=0$.

With this observation in mind we approximate $\Lambda_{\gamma}-\Lambda_{0}$ by using Dirichlet data of the form $f(\cdot)=\frac{\partial G}{\partial \nu_{x}}(\cdot, y), y \in \Gamma_{i}$, and solving the integral equations (3.1) and (3.7). Note that $G$ is a quite particular fundamental solution, namely the solution to (4.9). Practically it is very important that these normal derivatives can be calculated efficiently. To understand how, let $\widetilde{G}$ be the Green's function for $U$, that is, for each $x \in U$, let $\widetilde{G}(x, y)$ be the solution to

$$
\begin{cases}\Delta_{y} \widetilde{G}(x, y)=-\delta_{x}(y) & \text { in } U, \\ \widetilde{G}(x, y)=0 & y \in \Gamma_{e} .\end{cases}
$$


Since $U$ is a disk, $\widetilde{G}(x, y)$ has a simple explicit expression. In fact, $\widetilde{G}$ is given by

$$
\widetilde{G}(x, y)=-\frac{1}{2 \pi} \ln |x-y|+\frac{1}{2 \pi} \ln \left|\frac{r_{e}}{|x|} x-\frac{|x|}{r_{e}} y\right| .
$$

For any $y \in \Gamma_{i}$ we may now compute $\frac{\partial G}{\partial \nu_{x}}(\cdot, y)$ on $\Gamma_{e}$ by solving the integral equation (5.22) of the following lemma.

Lemma 5.6 For any $y \in \Gamma_{i}$, let $G_{y}(x):=G(x, y)$, and $\widetilde{G}_{y}(x):=\widetilde{G}(x, y)$ for $x \in \Gamma_{e}$. Then

$$
\left(I+2 \mathcal{K}_{\Gamma_{e}}^{*}-4 \frac{\partial \mathcal{S}_{\Gamma_{i}}}{\partial \nu_{e}} \frac{\partial \mathcal{S}_{\Gamma_{e}}}{\partial \nu_{i}}\right)\left[\frac{\partial G_{y}}{\partial \nu_{e}}\right](x)=2 \frac{\partial \widetilde{G}_{y}}{\partial \nu_{e}}(x), \quad x \in \Gamma_{e}
$$

where $\nu_{e}$ and $\nu_{i}$ denote outward normal vectors to $U$ on $\Gamma_{e}$, and to $D$ on $\Gamma_{i}$, respectively.

Proof. Fix $y \in \Omega$ and let $u(x)=\left(G_{y}-\widetilde{G}_{y}\right)(x)$ for $x \in \Omega$. Because of the symmetry of $G$ and $\widetilde{G}, u$ satisfies $\Delta u=0$ in $\Omega, u=0$ on $\Gamma_{e}$, and $\frac{\partial u}{\partial \nu_{i}}=-\frac{\partial \widetilde{G}_{y}}{\partial \nu_{i}}$ on $\Gamma_{i}$. Thus by the Green theorem, we have

$$
u(x)=-\mathcal{S}_{\Gamma_{e}}\left[\frac{\partial u}{\partial \nu_{e}}\right](x)+\mathcal{D}_{\Gamma_{i}}[u](x)+\mathcal{S}_{\Gamma_{i}}\left[\frac{\partial u}{\partial \nu_{i}}\right](x), \quad x \in \Omega
$$

However, in view of (2.3), we have

$$
\mathcal{D}_{\Gamma_{i}}[u](x)=\mathcal{S}_{\Gamma_{i}}\left(\frac{1}{2} I+\mathcal{K}_{\Gamma_{i}}^{*}\right)^{-1}\left[\left.\frac{\partial}{\partial \nu_{i}} \mathcal{D}_{\Gamma_{i}}[u]\right|_{\Gamma_{i}}\right](x)
$$

for all $x \in \mathbb{R}^{2} \backslash \bar{D}$. Therefore there is a density function $\psi$ with $\int_{\Gamma_{i}} \psi=0$ such that

$$
\left(G_{y}-\widetilde{G}_{y}\right)(x)=-\mathcal{S}_{\Gamma_{e}}\left[\frac{\partial G_{y}}{\partial \nu_{e}}-\frac{\partial \widetilde{G}_{y}}{\partial \nu_{e}}\right](x)+\mathcal{S}_{\Gamma_{i}}[\psi](x), \quad x \in \Omega .
$$

Similarly to (3.1) we now obtain from (5.23)

$$
\left(\frac{1}{2} I+\mathcal{K}_{\Gamma_{i}}^{*}\right)[\psi]=-\frac{\partial \widetilde{G}_{y}}{\partial \nu_{i}}+\frac{\partial \mathcal{S}_{\Gamma_{e}}}{\partial \nu_{i}}\left[\frac{\partial G_{y}}{\partial \nu_{e}}-\frac{\partial \widetilde{G}_{y}}{\partial \nu_{e}}\right] \quad \text { on } \Gamma_{i} .
$$

Since $\Gamma_{i}$ is a circle, $\mathcal{K}_{\Gamma_{i}}^{*}[\varphi]=0$ when $\int_{\Gamma_{i}} \varphi=0($ cf. (2.6)) and hence

$$
\psi=-2 \frac{\partial \widetilde{G}_{y}}{\partial \nu_{i}}+2 \frac{\partial \mathcal{S}_{\Gamma_{e}}}{\partial \nu_{i}}\left[\frac{\partial G_{y}}{\partial \nu_{e}}-\frac{\partial \widetilde{G}_{y}}{\partial \nu_{e}}\right] .
$$

Since $\widetilde{G}_{y}(x)+\mathcal{S}_{\Gamma_{e}}\left[\frac{\partial \widetilde{G}_{y}}{\partial \nu_{e}}\right](x)=-\Phi(x, y)$ for $x \in U$ and $y \in U$ (cf. Equation (2.23) of [1]), it follows that

$$
\psi=2 \frac{\partial \mathcal{S}_{\Gamma_{e}}}{\partial \nu_{i}}\left[\frac{\partial G_{y}}{\partial \nu_{e}}\right]+2 \frac{\partial \Phi_{y}}{\partial \nu_{i}}
$$


By substituting (5.24) into (5.23) and taking normal derivatives $\left(\right.$ on $\Gamma_{e}$ ) of both sides of (5.23), we obtain

$$
\left(\frac{1}{2} I+\mathcal{K}_{\Gamma_{e}}^{*}-2 \frac{\partial \mathcal{S}_{\Gamma_{i}}}{\partial \nu_{e}} \frac{\partial \mathcal{S}_{\Gamma_{e}}}{\partial \nu_{i}}\right)\left[\frac{\partial G_{y}}{\partial \nu_{e}}\right]=\left(\frac{1}{2} I+\mathcal{K}_{\Gamma_{e}}^{*}\right)\left[\frac{\partial \widetilde{G}_{y}}{\partial \nu_{e}}\right]+2 \frac{\partial \mathcal{S}_{\Gamma_{i}}}{\partial \nu_{e}}\left[\frac{\partial \Phi_{y}}{\partial \nu_{i}}\right] \quad \text { on } \Gamma_{e},
$$

for any fixed $y \in \Omega$. We claim that in the limit as $y$ approaches $\Gamma_{i}$ from inside $\Omega$

$$
\lim \left(\left(\frac{1}{2} I+\mathcal{K}_{\Gamma_{e}}^{*}\right)\left[\frac{\partial \widetilde{G}_{y}}{\partial \nu_{e}}\right]+2 \frac{\partial \mathcal{S}_{\Gamma_{i}}}{\partial \nu_{e}}\left[\frac{\partial \Phi_{y}}{\partial \nu_{i}}\right]\right)=\frac{\partial \widetilde{G}_{y}}{\partial \nu_{e}}
$$

and (5.22) immediately follows. It remains to verify (5.25). First of all, by (2.6),

$$
\begin{aligned}
\mathcal{K}_{\Gamma_{e}}^{*}\left[\frac{\partial \widetilde{G}_{y}}{\partial \nu_{e}}\right] & =\frac{1}{4 \pi r_{e}} \int_{\Gamma_{e}} \frac{\partial \widetilde{G}_{y}}{\partial \nu_{e}}(x) d s \\
& =\frac{1}{4 \pi r_{e}} \int_{U} \Delta \widetilde{G}_{y}(x) d x=-\frac{1}{4 \pi r_{e}}
\end{aligned}
$$

for any $y \in \Omega$. Secondly, in the limit as $y$ approaches $\Gamma_{i}$ from inside $\Omega$, we have

$$
\lim \mathcal{S}_{\Gamma_{i}}\left[\frac{\partial \Phi_{y}}{\partial \nu_{i}}\right](x)=-\frac{1}{4 \pi}[\ln |x-y|-\ln |x|], \quad \text { for }|x|>r_{i} .
$$

In fact, we have

$$
\mathcal{S}_{\Gamma_{i}}\left[\frac{\partial \Phi_{y}}{\partial \nu_{i}}\right](x)=\frac{1}{4 \pi^{2}} \int_{\Gamma_{i}} \ln |x-z| \frac{\partial}{\partial \nu_{z}} \ln |z-y| d s(z)=\mathcal{D}_{\Gamma_{i}}\left[\Phi_{x}\right](y),
$$

and hence from (2.4) and (2.6)

$$
\begin{aligned}
\lim \mathcal{S}_{\Gamma_{i}}\left[\frac{\partial \Phi_{y}}{\partial \nu_{i}}\right](x) & =-\frac{1}{2} \Phi_{x}(y)+\frac{1}{4 \pi r_{i}} \int_{\Gamma_{i}} \Phi_{x}(y) d \sigma(y) \\
& =-\frac{1}{4 \pi}[\ln |x-y|-\ln |x|]
\end{aligned}
$$

which is the desired formula. Note that the last equality holds because of (2.7).

A similar statement holds for any derivative with respect to $x,|x|>r_{i}$. In view of (5.21), it is now a matter of straight-forward computation to check that in the limit as $y$ approaches $\Gamma_{i}$ from inside $\Omega$

$$
\lim \left(\mathcal{K}_{\Gamma_{e}}^{*}\left[\frac{\partial \widetilde{G}_{y}}{\partial \nu_{e}}\right]+2 \frac{\partial \mathcal{S}_{\Gamma_{i}}}{\partial \nu_{e}}\left[\frac{\partial \Phi_{y}}{\partial \nu_{i}}\right]\right)=\frac{1}{2} \frac{\partial \widetilde{G}_{y}}{\partial \nu_{e}} \quad \text { on } \Gamma_{e},
$$

and we obtain (5.25). This completes the proof.

For computation, we discretize $\Gamma_{e}$ and $\Gamma_{i}$ by choosing points

$$
\left\{r_{e}\left(\cos \theta_{n}, \sin \theta_{n}\right) \mid \theta_{n}=2 \pi(n-1) / N, n=0,1, \ldots, N-1\right\}
$$


and

$$
\left\{r_{i}\left(\cos \theta_{n}, \sin \theta_{n}\right) \mid \theta_{n}=2 \pi(n-1) / N, n=0,1, \ldots, N-1\right\},
$$

with $N=256$. Put $x_{n}:=r_{e}\left(\cos \theta_{n}, \sin \theta_{n}\right)$ and $y_{n}:=r_{i}\left(\cos \theta_{n}, \sin \theta_{n}\right)$ for $n=0,1, \ldots, 255$. The discrete data we use for our numerical algorithm consist of the $256 \times 256$ matrix $\left(\left(\Lambda_{\gamma}-\Lambda_{0}\right)\left(\frac{\partial G}{\partial \nu_{x}}\left(\cdot, y_{n}\right)\right)\left(x_{m}\right)\right)_{m, n=1}^{256}$, or rather its SVD (singular value decomposition). For comparison we also calculate the $256 \times 256$ matrix $\left(T\left(\frac{\partial G}{\partial \nu}\left(\cdot, y_{n}\right)\right)\left(x_{m}\right)\right)_{m, n=1}^{256}$ and its SVD. Notice than none of these two matrices are symmetric.

Figure 1 displays the singular values of our discrete versions of $\Lambda_{\gamma}-\Lambda_{0}$ and $T$ when there are two internally corroded parts. The singular values of our approximation to $T$ exhibit a significant drop, to machine epsilon, after the first two (consistent with the fact that the range of $T$ is two-dimensional) there is also a drop in the singular values of our approximation to $\Lambda_{\gamma}-\Lambda_{0}$, but it is of course not nearly as significant due to the discrepancy between $\Lambda_{\gamma}-\Lambda_{0}$ and $T$, and due to the very approximate nature of our discrete data. It is nonetheless still possible to estimate the location of the corroded parts very accurately from the SVD of our discrete version of $\Lambda_{\gamma}-\Lambda_{0}$.

Using the first $k$ (left-)singular vectors of our data, we plot approximate values of $\theta_{k}^{\epsilon}(z)=$ $\cot ^{-1} L_{k}^{\epsilon}(z)$ where

$$
L_{k}^{\epsilon}(z):=\frac{\left\|P_{k}^{\epsilon}\left(\frac{\partial G(\cdot, z)}{\partial \nu}\right)\right\|_{L^{2}\left(\Gamma_{e}\right)}}{\left\|\left(I-P_{k}^{\epsilon}\right)\left(\frac{\partial G(\cdot, z)}{\partial \nu}\right)\right\|_{L^{2}\left(\Gamma_{e}\right)}} .
$$

We start this process with just one singular vector $(k=1)$ and continue with increasing $k$ until the plot of $\theta_{k}^{\epsilon}(z)$ stabilizes. Values near zero correspond to locations are that likely to be close to the $I_{s}$ (or $z_{s}$ ).

Example 1. In this example, the outer radius $r_{e}=1$ and the inner one $r_{i}=0.8$ and there are two corroded parts. The top part of Figure 1 shows the locations of the corrosion and the approximate SVD of $\Lambda_{\gamma}-\Lambda_{0}$ and $T$. The bottom of Figure 1 clearly shows that there are two corroded parts, since the minima of $\theta_{k}^{\epsilon}$ stabilize already at $k=2$. The minima of $\theta_{2}^{\epsilon}$ are the locations of the corroded parts. Since we use the same 256 points for the calculation and plotting of $\theta_{k}^{\epsilon}$ and for the potential location of the points $z_{s}$, the fact that $\left|z_{s}-z_{s}^{c}\right|=0$ really asserts that $z_{s}$ is accurately estimated up to the spacing between two adjacent mesh points, which is approximately 0.0172 . By solving (5.19), we compute the integrated corrosion coefficients: $\langle\gamma\rangle_{1}^{c}=0.3846,\langle\gamma\rangle_{2}^{c}=0.1660$. The actual, integrated coefficients are $\langle\gamma\rangle_{1}=0.3927,\langle\gamma\rangle_{2}=0.1767$.

Example 2. In this example we consider a case with five corroded parts. The actual data of the configuration is summarized in the top of Table 1. The corroded parts are numbered counter-clock-wise starting with the smallest positive angle. Note that the first three corroded parts have low corrosion coefficients while the remaining two have relatively high ones. The computational results with $1 \%$ noise are summarized in Table 1 and Figure 2. It is interesting to note that the corroded parts 1 and 2 which are close to each other and have low corrosion coefficients, are detected as a single one. The actual midpoint locations of corroded parts 1 and 2 are $(0.0686,0.6966)$ and $(-0.1197,0.6897)$, and the single detected location is $(-0.1027,0.6924)$. On the other hand, the corroded parts 4 and 5 , which have high corrosion coefficients, are clearly detected already with $k=3$. 
actual data

\begin{tabular}{cccc}
\multicolumn{4}{c}{ actual data } \\
\hline$m$ & $\gamma_{s}$ & $z_{s}$ & $\langle\gamma\rangle_{s}$ \\
\hline 5 & 0.1 & $(0.0686,0.6966)$ & 0.0120 \\
& 0.5 & $(-0.1197,0.6897)$ & 0.0430 \\
& 0.3 & $(-0.4307,0.5518)$ & 0.0206 \\
& 1.0 & $(0.2519,-0.6531)$ & 0.1203 \\
& 1.2 & $(0.5723,-0.4031)$ & 0.1031 \\
\hline
\end{tabular}

\begin{tabular}{ccccc}
\multicolumn{5}{c}{ detected data } \\
\hline$m^{c}$ & $z_{s}^{c}$ & $\langle\gamma\rangle_{s}^{c}$ & $\left|z_{s}-z_{s}^{c}\right|$ & $\left|\langle\gamma\rangle_{s}-\langle\gamma\rangle_{s}^{c}\right|$ \\
\hline 4 & $(-0.1027,0.6924)$ & 0.0514 & 0.0172 & 0.0084 \\
& $(-0.4170,0.5622)$ & 0.0212 & 0.0172 & 0.0006 \\
& $(0.2519,-0.6531)$ & 0.1181 & 0 & 0.0021 \\
& $(0.5723,-0.4031)$ & 0.1026 & 0 & 0.0005 \\
\hline
\end{tabular}

result for each step

\begin{tabular}{|c|c|c|c|c|c|}
\hline $\begin{array}{c}\text { number of } \\
\text { sing. vectors }(k)\end{array}$ & $m^{c}$ & $z_{s}^{c}$ & $\left|z_{s}-z_{s}^{c}\right|$ & $\langle\gamma\rangle_{s}^{c}$ & $\left|\langle\gamma\rangle_{s}-\langle\gamma\rangle_{s}^{c}\right|$ \\
\hline 1 & 1 & $(0.3889,-0.5820)$ & & 0.1554 & \\
\hline \multirow[t]{2}{*}{2} & 2 & $(-0.1366,+0.6865)$ & & 0.0579 & \\
\hline & & $(0.3889,-0.5820)$ & & 0.1554 & \\
\hline \multirow[t]{3}{*}{3} & 3 & $(-0.1366,+0.6865)$ & & 0.0579 & \\
\hline & & $(0.2519,-0.6531)$ & & 0.1181 & \\
\hline & & $(0.5723,-0.4031)$ & & 0.1026 & \\
\hline \multirow[t]{4}{*}{4} & & $(-0.0686,+0.6966)$ & 0.0515 & 0.0486 & 0.0056 \\
\hline & & $(-0.4031,+0.5723)$ & 0.0344 & 0.0237 & 0.0030 \\
\hline & & $(0.2519,-0.6531)$ & 0 & 0.1181 & 0.0021 \\
\hline & & $(0.5723,-0.4031)$ & 0 & 0.1026 & 0.0005 \\
\hline \multirow[t]{4}{*}{5} & 4 & $(-0.1027,+0.6924)$ & 0.0172 & 0.0514 & 0.0084 \\
\hline & & $(-0.4170,+0.5622)$ & 0.0172 & 0.0212 & 0.0006 \\
\hline & & $(0.2519,-0.6531)$ & 0 & 0.1181 & 0.0021 \\
\hline & & $(0.5723,-0.4031)$ & 0 & 0.1026 & 0.0005 \\
\hline \multirow[t]{4}{*}{6} & 4 & $(-0.1027,+0.6924)$ & 0.0172 & 0.0514 & 0.0084 \\
\hline & & $(-0.4170,+0.5622)$ & 0.0172 & 0.0212 & 0.0006 \\
\hline & & $(0.2519,-0.6531)$ & 0 & 0.1181 & 0.0021 \\
\hline & & $(0.5723,-0.4031)$ & 0 & 0.1026 & 0.0005 \\
\hline
\end{tabular}

Table 1: Summary of computational results with five corroded parts, and $1 \%$ noise. Two corroded parts with low corrosion coefficient are detected as a single one. 

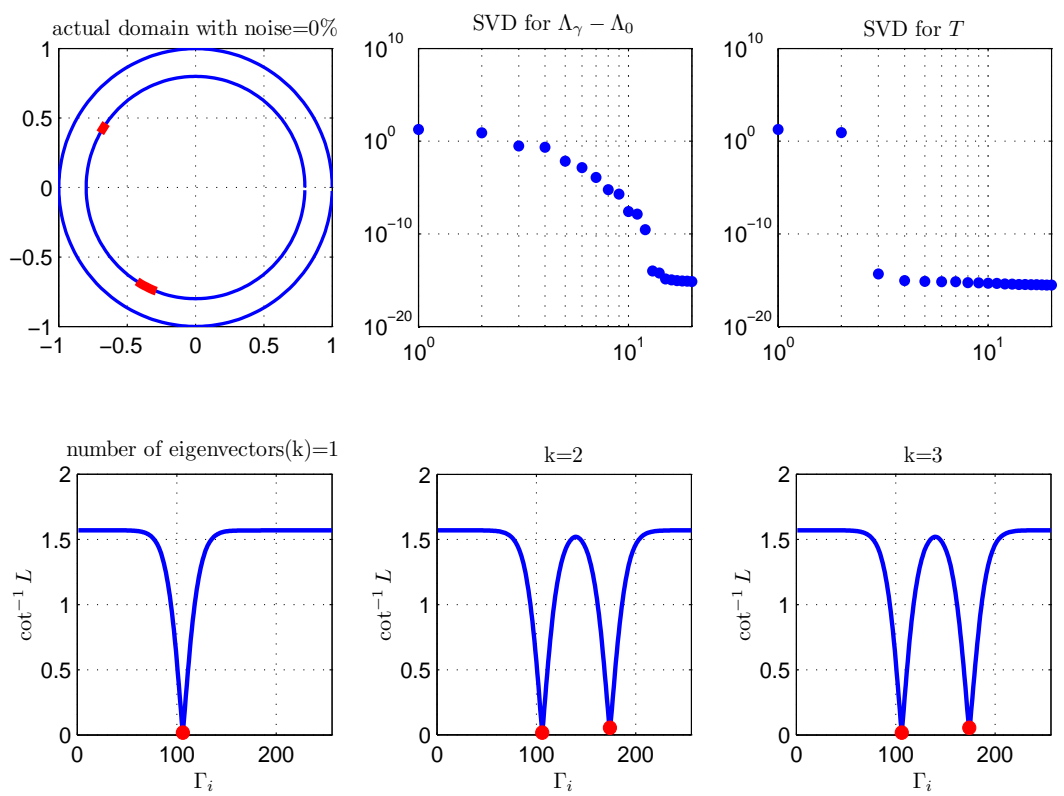

The results for steps

\begin{tabular}{cccccc}
\hline $\begin{array}{c}\text { number of } \\
\text { sing. vectors }(k)\end{array}$ & $m^{c}$ & $z_{s}^{c}$ & $\left|z_{s}-z_{s}^{c}\right|$ & $\langle\gamma\rangle_{s}^{c}$ & $\left|\langle\gamma\rangle_{s}-\langle\gamma\rangle_{s}^{c}\right|$ \\
\hline 1 & 1 & $(-0.6759,0.4280)$ & & 0.3846 & \\
\hline 2 & 2 & $(-0.6759,0.4280)$ & 0 & 0.3846 & 0.0081 \\
& & $(-0.3597,-0.7146)$ & 0 & 0.1660 & 0.0107 \\
\hline 3 & 2 & $(-0.6759,0.4280)$ & 0 & 0.3846 & 0.0081 \\
& & $(-0.3597,-0.7146)$ & 0 & 0.1660 & 0.0107 \\
\hline
\end{tabular}

Figure 1: Pipe with two internal corroded parts, and "approximate" singular values of $\Lambda_{\gamma}-\Lambda_{0}$ and $T$ in upper figures. The bottom figure shows $\theta_{k}^{\epsilon}$ for three different values of $k$. In the table, $m^{c}$ is the number of estimated corroded parts, $z_{s}$ and $z_{s}^{c}$ are the actual midpoint locations and the estimated midpoint locations, and $\langle\gamma\rangle_{s},\langle\gamma\rangle_{s}^{c}$ are the actual and the estimated, integrated, corrosion coefficients, for a particular $k$.

Example 3. Figure 3 shows the computational results with various degree of noise. The information about the actual locations and corrosion coefficients is summarized in Table 2. Observe that the first two corroded parts have low corrosion coefficients. They are detected as a single corroded part with $5 \%$ noise. The other two corroded parts, which have high corrosion coefficients, are detected very well even with a high ratio of noise.

\section{Conclusion}

We have designed a non-iterative algorithm of MUSIC-type for detecting small, internal, corroded boundary parts based on external, electrostatic boundary measurements. Our 

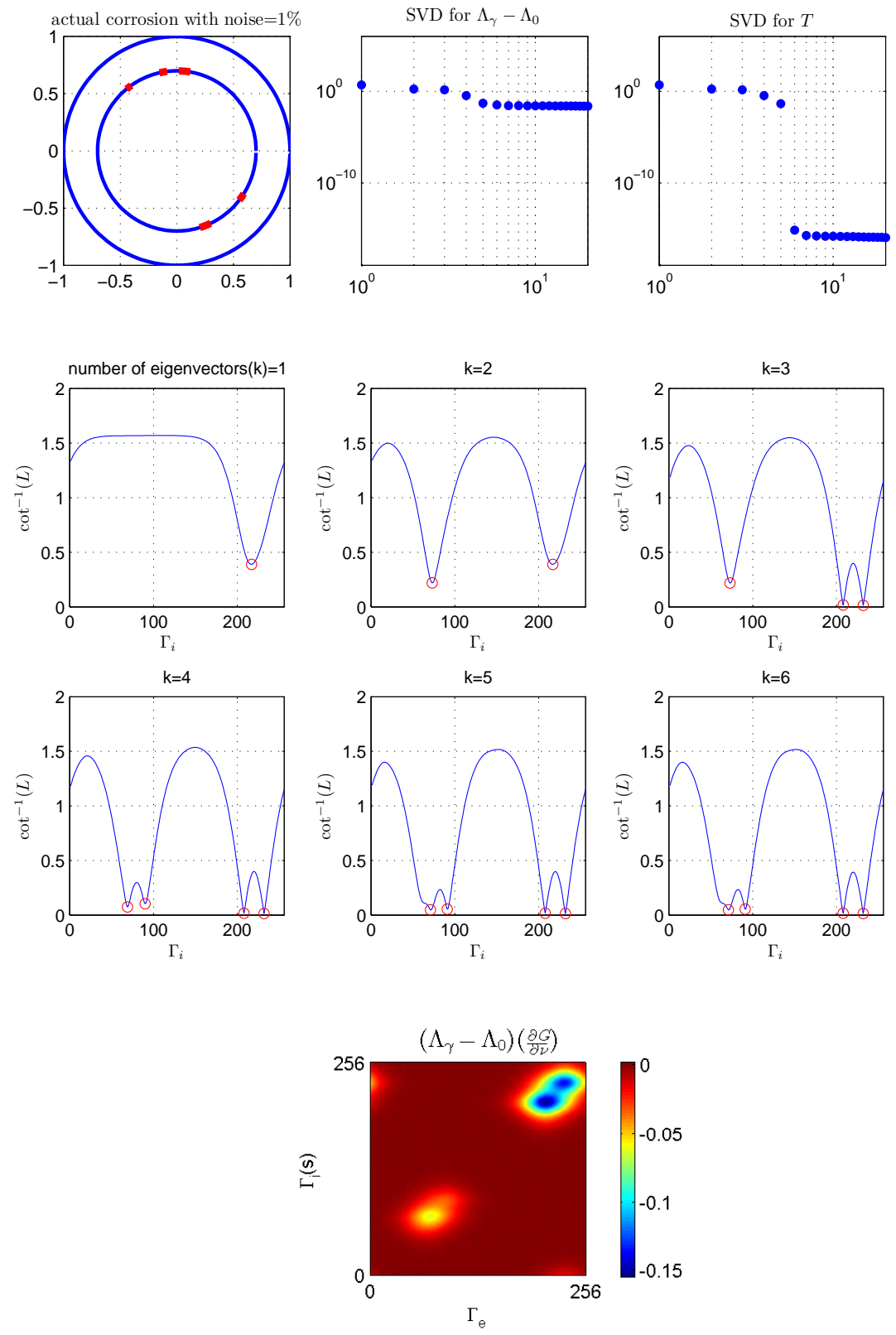

Figure 2: The computational results with $1 \%$ noise. The last plot shows the values of $\left(\Lambda_{\gamma}-\Lambda_{0}\right)\left(\frac{\partial G}{\partial \nu}\right)$ on $\Gamma_{e} \times \Gamma_{i}$.

method relies on an asymptotic representation formula for the steady state current perturbations caused by small, internal corroded boundary parts. We have performed numerical 


\begin{tabular}{cccc}
\hline$m$ & $\gamma_{s}$ & $z_{s}$ & $\langle\gamma\rangle_{s}$ \\
\hline 4 & 0.1 & $(0.5657,+0.5657)$ & 0.0137 \\
& 0.05 & $(0.3597,+0.7146)$ & 0.0049 \\
& 3.0 & $(0.6307,-0.4922)$ & 0.2945 \\
& 3.0 & $(0.7464,-0.2879)$ & 0.2945 \\
\hline
\end{tabular}

Table 2: Pipe with four corrosive parts.
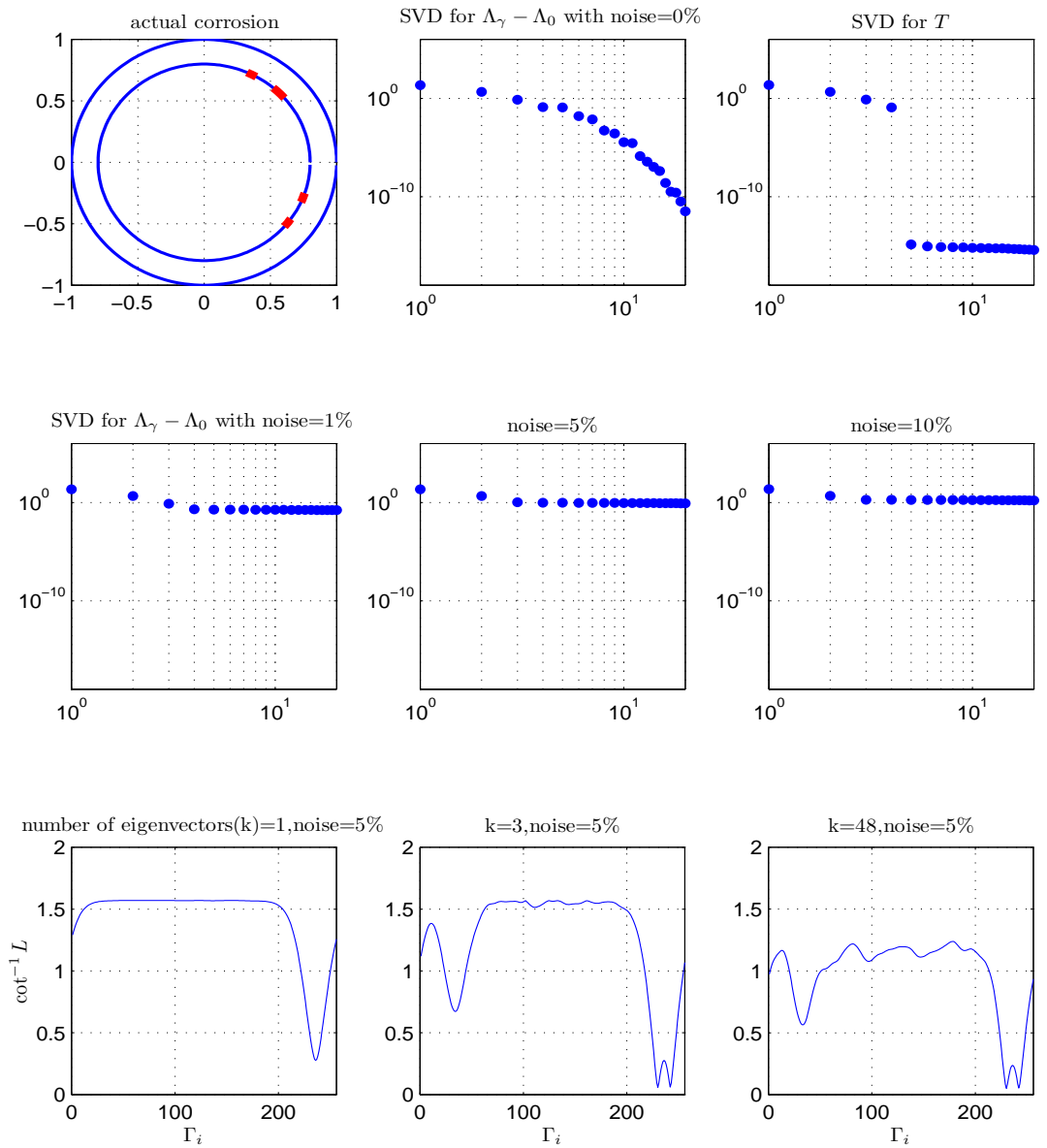

Figure 3: Computational results with varying degree of noise

experiments to test the viability of the algorithm using a discrete version of $\Lambda_{\gamma}-\Lambda_{0}$ as data. The numerical tests clearly demonstrate that the algorithm works well even in the presence of relatively high noise ratios, provided the corrosion coefficients are sufficiently large. The latter restriction is to be expected, since only those corroded parts with reasonably sized 
corrosion coefficients can cause sufficient changes in currents. Furthermore, it is practically most important to detect the corroded parts with the highest coefficients, since they are likely to "develop" more rapidly, and therefore potentially cause the most serious damage (to the pipe). It should be mentioned that our algorithm cannot identify separately the size of the corroded parts (without assuming that we know the corrosion coefficients). We can only reconstruct the integral $\langle\gamma\rangle_{s}=\int_{I_{s}} \gamma d \sigma$ for $s=1, \ldots, m$. It is likely that a higher-order asymptotic expansion of the boundary perturbations would yield formulas that permit such identification.

Acknowledgments. We would like to thank the referees for their careful reading of the manuscript and their many suggestions. We feel the implementation of some of these suggestions has made our presentation more accessible.

\section{References}

[1] H. Ammari and H. Kang, High-order terms in the asymptotic expansions of the steadystate voltage potentials in the presence of conductivity inhomogeneities of small diameter, SIAM J. Math. Anal., 34 (2003), 1152-1166.

[2] H. Ammari, S. Moskow, and M.S. Vogelius, Boundary integral formulas for the reconstruction of electromagnetic imperfections of small diameter, ESAIM: Cont. Opt. Calc. Var., 9 (2003), 49-66.

[3] H.T. Banks, M.L. Joyner, B. Wincheski, and W.P. Winfree, Real time computational algorithms for eddy-current-based damage detection, Inverse Problems, 18 (2002), 795823.

[4] M. Brühl, M. Hanke, and M.S. Vogelius, A direct impedance tomography algorithm for locating small inhomogeneities, Numer. Math., 93 (2003), 635-654.

[5] G. Buttazzo and R.V. Kohn, Reinforcement by a thin layer with oscillating thickness, Appl. Math. Opt., 16 (1988), 247-261.

[6] D.J. Cedio-Fengya, S. Moskow, and M.S. Vogelius, Identification of conductivity imperfections of small diameter by boundary measurements. Continuous dependence and computational reconstruction, Inverse Problems, 14 (1998), 553-595.

[7] M. Cheney, The linear sampling method and the MUSIC algorithm, Inverse Problems, 17 (2001), 591-595.

[8] R.R. Coifman, A. McIntosh, and Y. Meyer, L'intégrale de Cauchy définit un opérateur borné sur $L^{2}$ pour les courbes lipschitziennes, Ann. of Math. (2), 116 (1982), 361-387.

[9] D. Colton and A. Kirsch, A simple method for solving inverse scattering problems in the resonance region, Inverse Problems, 12 (1996), 383-393.

[10] A.J. Devaney, Time reversal imaging of obscured targets from multistatic data, IEEE Trans. Antennas Propagat., 523 (2005), 1600-1610.

[11] G.B. Folland, Introduction to Partial Differential Equations, Princeton University Press, Princeton, NJ, 1976. 
[12] G. Inglese, An inverse problem in corrosion detection, Inverse Problems, 13 (1997), 977-994.

[13] B. Luong and F. Santosa, Quantitative imaging of corrosion inplates by eddy current methods, SIAM J. Appl. Math., 58 (1998), 1509-1531.

[14] H. Kang and J.K. Seo, Layer potential technique for the inverse conductivity problem, Inverse Problems, 12 (1996), 267-278.

[15] - Recent progress in the inverse conductivity problem with single measurement, in Inverse Problems and Related Fields, CRC Press, Boca Raton, FL, 2000, $69-80$.

[16] P. Kaup and F. Santosa, Nondestructive evaluation of corrosion damage using electrostatic measurements, J. Nondestructive Eval. 14 (1995), 127-136.

[17] P. Kaup, F. Santosa, and M. Vogelius, A method for imaging corrosion damage in thin plates from electrostatic data, Inverse Problems, 12 (1996), 279-293.

[18] A. Kirsch, The MUSIC algorithm and the factorisation method in inverse scattering theory for inhomogeneous media, Inverse Problems, 18 (2002), 1025-1040.

[19] E.M. Stein, Singular Integrals and Differentiability Properties of Functions, Princeton University Press, Princeton, NJ, 1970.

[20] C.W. Therrien, Discrete Random Signals and Statistical Signal Processing, PrenticeHall, Englewood Cliffs, NJ, 1992.

[21] M. Vogelius and J. Xu, A nonlinear elliptic boundary value problem related to corrosion modelling, Quart. Appl. Math., 56 (1998), 479-505.

[22] X. Yang, M. Choulli, and J. Cheng, An iterative BEM for the inverse problem of detecting corrosion in a pipe, Numer. Math. J. Chinese Univ., 14 (2005), 252-266. 